\title{
Charged particles interaction in both a finite volume and a uniform magnetic field
}

\author{
Peng Guo $\odot^{1,2, *}$ and Vladimir Gasparian $\oplus^{1}$ \\ ${ }^{1}$ Department of Physics and Engineering, California State University, Bakersfield, California 93311, USA \\ ${ }^{2}$ Kavli Institute for Theoretical Physics, University of California, Santa Barbara, California 93106, USA
}

(Received 5 January 2021; accepted 10 May 2021; published 27 May 2021)

\begin{abstract}
A formalism for describing charged particles interaction in both a finite volume and a uniform magnetic field is presented. In the case of short-range interaction between charged particles, we show that the factorization between short-range physics and finite volume long-range correlation effect is possible; a Lüscher formulalike quantization condition is thus obtained.
\end{abstract}

DOI: 10.1103/PhysRevD.103.094520

\section{INTRODUCTION}

In recent years, a great effort in the nuclear and hadron physics community has been put into constructing the scattering dynamics of few-particle interactions from the discrete bound state energy spectrum that is computed in various types of traps, such as the commonly used periodic finite box in lattice QCD (LQCD) and the harmonic oscillator trap in nuclear physics computation. The ultimate goal is of course to study and explore the nature of particle interactions that plays an essential role in many fields of physical science, such as nuclear physics and astrophysics. However, the current state-of-art $a b$ initio computations in nuclear and hadron physics are normally performed in a harmonic oscillator trap and in a finite volume respectively. Instead of computing few-body scattering amplitudes, the discrete bound state energy levels are usually directly measured and extracted from these ab initio computations. Therefore, finding a relation that converts a discrete bound state energy spectrum into a continuum scattering state is a key step.

In fact, relating the energy shift caused by particle interactions to the on-shell scattering parameters such as phase shift has a long history across many fields in physics. In general cases, the dynamics of particles interaction in traps is associated with the infinite volume off-shell reaction amplitudes in a highly nontrivial way. Fortunately, when the separation of two physics scales, the size of trap and the range of particles interaction, is clearly established, a simple asymptotic form can be found,

pguo@csub.edu

Published by the American Physical Society under the terms of the Creative Commons Attribution 4.0 International license. Further distribution of this work must maintain attribution to the author(s) and the published article's title, journal citation, and DOI. Funded by SCOAP. which provides a relation between energy levels in a trap and the infinite volume scattering phase shift. In finite volume in LQCD computation, such a relation in the elastic two-body sector is known as the Lüscher formula [1], which shows a clear factorization of short-range dynamics and long-range correlation effects because of the periodic boundary condition. The short-range dynamics and longrange correlations are described by the physical scattering phase shift and Lüscher's zeta function respectively. Lüscher's formula has been proving very successful in the LQCD community, and it has been quickly extended into both coupled-channel and few-body sectors, see [2-41]. In nuclear physics where a harmonic oscillator trap is commonly used, such a relation is given by the Busch-Englert-Rzażewski-Wilkens (BERW) formula [4253]. In addition to the periodic boundary condition and harmonic trap, other types of traps or boundary conditions are also commonly used in different physics fields, such as the hard wall trap [54,55]. Regardless of the difference among various traps, the same strategy is shared: as the two physical scales are clearly separated, a closed asymptotic form can be found, in which short-range dynamics is described by a scattering phase shift and the long-range effect is given by an analytic form that describes how the propagation of particles is affected by the trap, e.g., Lüscher's zeta function in a periodic boundary condition.

In the present work, we aim to establish a similar relation to Lüscher's and the BERW formula for the charged particles interacting in both a uniform magnetic field and a periodic box. We remark that only a short-range interaction which represents nuclear force or hadron interactions is considered in this work; the Coulomb interaction has not been incorporated in the current framework yet. We also emphasize that the Coulomb repulsion may be important near threshold [56-67], especially since the long-range nature of the Coulomb interaction may complicate the factorization of physics at different scales and distort the 
asymptotic wave functions, the Coulomb interaction must be included in future work. We will show that with only a short-range potential, the factorization of short-range physics and the long-range correlation effect is possible. Hence a relation in a compact form that relates the discrete energy spectrum to scattering phase shifts can be found. Such a relation may be useful for the study of a charged hadron system such as the $\pi^{+}$system in LQCD computation. In finite volume, in order to preserve translation symmetry of a system in a magnetic field, the magnetic flux through a perpendicular surface of a cubic box to a uniform magnetic field must be $2 \pi$ multiplied by a rational number $n_{p} / n_{q}$, where $n_{p}$ and $n_{q}$ are integers and relatively prime to each other. Therefore, the original energy level without a magnetic field is split into $n_{q}$ sublevels due to the application of the magnetic field. We also remark that the ultimate goal of the current work is to set up a foundation for exploring the possibility of the topological edge type states [68-70] in lattice QCD in future work. However, by using background-field methods in lattice QCD [71-74], the finite volume energy levels of particles interacting in a magnetic field background may also be used to determine the coefficient of the leading local fournucleon operator contributing to the neutral- and chargedcurrent breakup of the deuteron.

The paper is organized as follows. The general formalism of charged bosons interaction in both a finite volume and a uniform magnetic field is presented in details in Sec. II. The $S$-wave contribution and regularization of ultraviolet divergence are discussed in Sec. III. A summary is given in Sec. IV.

\section{FINITE VOLUME DYNAMICS OF CHARGED BOSONS IN A UNIFORM MAGNETIC FIELD}

In this section, we briefly summarize the dynamics of charged bosons interacting in both a finite periodic box and a uniform magnetic field. The uniform magnetic field is chosen along the $z$ axis, $\mathbf{B}=B \mathbf{e}_{z}$, and the Landau gauge for the vector potential is adopted in this work,

$$
\mathbf{A}(\mathbf{x})=B(0, x, 0)
$$

The complete presentation and more rigorous discussion are given in the Appendixes A and B.

The dynamics of the relative motion of two charged identical nonrelativistic spinless particles in a uniform magnetic field is described by the Schrödinger equation,

$$
\left(\hat{H}_{\mathbf{r}}+V(\mathbf{r})\right) \psi_{\varepsilon}(\mathbf{r})=\varepsilon \psi_{\varepsilon}(\mathbf{r}),
$$

where $\psi_{\varepsilon}(\mathbf{r})$ and $\varepsilon$ are the wave function and energy for the relative motion of a two charged boson system. The Hamiltonian operator $\hat{H}_{\mathbf{r}}$ is defined by

$$
\hat{H}_{\mathbf{r}}=-\frac{\left(\nabla_{\mathbf{r}}+i q \mathbf{A}(\mathbf{r})\right)^{2}}{2 \mu}
$$

where $\mu$ and $q$ denote the reduced mass and charge of two charged particles respectively.

\section{A. Magnetic periodic boundary condition}

In a periodic finite box, the discrete translation invariance is broken by a coordinate dependent vector potential,

$$
A_{y}(\mathbf{r}+\mathbf{n} L)=A_{y}(\mathbf{r})+B \mathbf{n} L \cdot \mathbf{e}_{x} .
$$

In order to maintain the discrete translation symmetry in a uniform magnetic field, the conventional translation operator must be generalized to the magnetic translation operator,

$$
\hat{T}_{\mathbf{r}}(\mathbf{n} L)=e^{i\left(-i \nabla_{\mathbf{r}}+q \mathbf{A}(\mathbf{r})-q \mathbf{B} \times \mathbf{r}\right) \cdot \mathbf{n} L} .
$$

Hence

$$
\hat{T}_{\mathbf{r}}(\mathbf{n} L) \psi_{\varepsilon}(\mathbf{r})=e^{i q B r_{y} \mathbf{e}_{x} \cdot \mathbf{n} L} \psi_{\varepsilon}(\mathbf{r}+\mathbf{n} L) .
$$

In addition, to warrant a state remain translational invariant through a closed path, the magnetic flux through an enlarged closed path in an $x-y$ plane that is defined by magnetic unit vectors

$$
n_{q} L \mathbf{e}_{x} \times L \mathbf{e}_{y} \times L \mathbf{e}_{z},
$$

must be quantized, see Refs. [75,76],

$$
q B n_{q} L^{2}=2 \pi n_{p},
$$

where $n_{p}$ and $n_{q}$ are two relatively prime integers. Therefore, the discrete translation in a enlarged magnetic unit box leaves the Hamiltonian invariant, and $\hat{T}_{\mathbf{r}}\left(\mathbf{n}_{B} L\right)$ forms a magnetic translation group, where

$$
\mathbf{n}_{B}=n_{x} n_{q} \mathbf{e}_{x}+n_{y} \mathbf{e}_{y}+n_{z} \mathbf{e}_{z}, \quad n_{x, y, z} \in \mathbb{Z} .
$$

According to Bloch's theorem, in a periodic box, periodicity of a system requires that $\hat{T}_{\mathbf{r}}\left(\mathbf{n}_{B} L\right) \psi_{\varepsilon}(\mathbf{r})$ can only differ from $\psi_{\varepsilon}(\mathbf{r})$ by a phase factor, which can be chosen as

$$
e^{i \frac{\mathbf{P}_{B}}{2} \cdot \mathbf{n}_{B} L}
$$

where

$$
\mathbf{P}_{B}=\frac{2 \pi}{L}\left(\frac{n_{x}}{n_{q}} \mathbf{e}_{x}+n_{y} \mathbf{e}_{y}+n_{z} \mathbf{e}_{z}\right), \quad n_{x, y, z} \in \mathbb{Z} .
$$

Hence the magnetic periodic boundary condition is given by 


$$
\psi_{\varepsilon}\left(\mathbf{r}+\mathbf{n}_{B} L\right)=e^{i \frac{\mathbf{P}_{B}}{2} \cdot \mathbf{n}_{B} L} e^{-i q B r_{y} \mathbf{e}_{x} \cdot \mathbf{n}_{B} L} \psi_{\varepsilon}(\mathbf{r}) .
$$

The magnetic periodic boundary condition can also be obtained by considering a separable form of a total wave function, see Appendix A 2.

\section{B. Finite volume Lippmann-Schwinger equation and quantization condition}

\section{Finite volume Lippmann-Schwinger equation}

The Schrödinger equation and magnetic periodic boundary condition in Eq. (2) and Eq. (9) together can be replaced by the finite volume homogeneous LippmannSchwinger (LS) equation,

$$
\psi_{\varepsilon}(\mathbf{r})=\int_{L_{B}^{3}} d \mathbf{r}^{\prime} G_{B}^{(L)}\left(\mathbf{r}, \mathbf{r}^{\prime} ; \varepsilon\right) V\left(\mathbf{r}^{\prime}\right) \psi_{\varepsilon}\left(\mathbf{r}^{\prime}\right),
$$

where the volume integration over the magnetic unit cell is defined by

$$
\int_{L_{B}^{3}} d \mathbf{r}^{\prime}=\int_{-\frac{n_{q} L}{2}}^{\frac{n_{q} L}{2}} d r_{x}^{\prime} \int_{-\frac{L}{2}}^{\frac{L}{2}} d r_{y}^{\prime} \int_{-\frac{L}{2}}^{\frac{L}{2}} d r_{z}^{\prime} .
$$

The finite volume magnetic Green's function $G_{B}^{(L)}$ satisfies the dynamical equation

$$
\begin{aligned}
(\varepsilon & \left.-\hat{H}_{\mathbf{r}}\right) G_{B}^{(L)}\left(\mathbf{r}, \mathbf{r}^{\prime} ; \varepsilon\right) \\
& =\sum_{\mathbf{n}_{B}} e^{-i \frac{\mathbf{P}_{B}}{2} \cdot \mathbf{n}_{B} L} e^{i q B r_{y} \mathbf{e}_{x} \cdot \mathbf{n}_{B} L} \delta\left(\mathbf{r}-\mathbf{r}^{\prime}+\mathbf{n}_{B} L\right) .
\end{aligned}
$$

The solution of a finite volume magnetic Green's function $G_{B}^{(L)}$ can be constructed from its infinite volume counterpart $G_{B}^{(\infty)}$ by

$$
\begin{aligned}
& G_{B}^{(L)}\left(\mathbf{r}, \mathbf{r}^{\prime} ; \varepsilon\right) \\
& =\sum_{\mathbf{n}_{B}} G_{B}^{(\infty)}\left(\mathbf{r}, \mathbf{r}^{\prime}+\mathbf{n}_{B} L ; \varepsilon\right) e^{i \frac{\mathbf{P}_{B}}{2} \cdot \mathbf{n}_{B} L} e^{-i q B r_{y}^{\prime} \mathbf{e}_{x} \cdot \mathbf{n}_{B} L} \\
& =\sum_{\mathbf{n}_{B}} e^{-i \frac{\mathbf{P}_{B}}{2} \cdot \mathbf{n}_{B} L} e^{i q B r_{y} \mathbf{e}_{x} \cdot \mathbf{n}_{B} L} G_{B}^{(\infty)}\left(\mathbf{r}+\mathbf{n}_{B} L, \mathbf{r}^{\prime} ; \varepsilon\right) .
\end{aligned}
$$

Details of the construction can be found in Appendix A 4. The analytic expression of an infinite volume magnetic Green's function $G_{B}^{(\infty)}$ is given by

$$
\begin{aligned}
G_{B}^{(\infty)}\left(\mathbf{r}, \mathbf{r}^{\prime} ; \varepsilon\right)= & -\frac{2 \mu q B}{2 \pi} e^{-\frac{i q B}{2}\left(r_{x}+r_{x}^{\prime}\right)\left(r_{y}-r_{y}^{\prime}\right)} e^{-\frac{q B}{4}\left|\boldsymbol{\rho}-\boldsymbol{\rho}^{\prime}\right|^{2}} \\
& \times \sum_{n=0}^{\infty} \frac{i L_{n}\left(\frac{q B}{2}\left|\boldsymbol{\rho}-\boldsymbol{\rho}^{\prime}\right|^{2}\right) e^{i \sqrt{2 \mu \varepsilon-2 q B\left(n+\frac{1}{2}\right)}\left|r_{z}-r_{z}^{\prime}\right|}}{2 \sqrt{2 \mu \varepsilon-2 q B\left(n+\frac{1}{2}\right)}}
\end{aligned}
$$

where $L_{n}(x)$ is the Laguerre polynomial, and

$$
\boldsymbol{\rho}=r_{x} \mathbf{e}_{x}+r_{y} \mathbf{e}_{y}, \quad \boldsymbol{\rho}^{\prime}=r_{x}^{\prime} \mathbf{e}_{x}+r_{y}^{\prime} \mathbf{e}_{y}
$$

are relative coordinates defined in the $x-y$ plane.

\section{Quantization condition with short-range interaction}

The discrete bound state energy spectrum can be found as the eigen-energy solutions of the homogeneous LS equation in Eq. (10). The partial wave expansion in an angular momentum basis is commonly used in describing an infinite volume scattering state. However in a magnetic field, due to the asymmetry of the magnetic Hamiltonian in $x-y$ plane and along the $z$ axis, the angular momentum basis in spherical coordinates is in fact not the most convenient basis in describing dynamics of charged particles in a uniform magnetic field. Nevertheless, it can be done in principle. For the sake of the consistency of presentation in both finite volume and infinite volume dynamics, let us consider the partial wave expansion of Eq. (10), which yields

$\psi_{l m}^{(L)}(r)=\sum_{l^{\prime} m^{\prime}} \int_{L_{B}^{3}} r^{\prime 2} d r^{\prime} G_{l m, l^{\prime} m^{\prime}}^{(B, L)}\left(r, r^{\prime} ; \varepsilon\right) V_{l^{\prime}}\left(r^{\prime}\right) \psi_{l^{\prime} m^{\prime}}^{(L)}\left(r^{\prime}\right)$.

The purpose of this work is to find a Lüscher formulalike simple relation that connects short-range physics associated with particles interaction $V(\mathbf{r})$ and the long-range effect generated by the finite volume and magnetic field. Also consider the fact that such a relation is the result of clear separation of two physical scales: (1) the range of potential $V(\mathbf{r})$ and (2) the size of a trap or finite volume. When the two scales are clearly separated, the short- and long-range physics can be factorized, and a compact relation as the leading order contribution can be found by studying the asymptotic behavior of the wave function [8,24]. Therefore, for our purpose, it is sufficient to consider the zero-range potential,

$$
V_{l}(r) \rightarrow V_{l} \frac{\delta(r)}{r^{2}} \frac{2^{2 l+1} \Gamma^{2}\left(l+\frac{3}{2}\right)}{(2 \pi)^{3} r^{2 l}},
$$

see Appendix B for a more rigorous discussion. Equation (15) is thus turned into an algebraic equation,

$$
\begin{aligned}
\frac{\psi_{l m}^{(L)}(r)}{r^{l}}= & \sum_{l^{\prime} m^{\prime}} V_{l^{\prime}} \frac{2^{2 l^{\prime}+1} \Gamma^{2}\left(l^{\prime}+\frac{3}{2}\right)}{(2 \pi)^{3}} \\
& \times\left.\frac{G_{l m, l^{\prime} m^{\prime}}^{(B, L)}\left(r, r^{\prime} ; \varepsilon\right)}{r^{l} r^{\prime l^{\prime}}} \frac{\psi_{l^{\prime} m^{\prime}}^{(L)}\left(r^{\prime}\right)}{r^{\prime l^{\prime}}}\right|_{r^{\prime} \rightarrow 0} .
\end{aligned}
$$

Hence the quantization condition of the discrete energy spectrum is given by 
$\operatorname{det}\left[\frac{\delta_{l m, l^{\prime} m^{\prime}}}{2^{2 l+1} V_{l}}-\left.\frac{\Gamma^{2}\left(l^{\prime}+\frac{3}{2}\right)}{(2 \pi)^{3}} \frac{G_{l m, l^{\prime} m^{\prime}}^{(B, L)}\left(r, r^{\prime} ; \varepsilon\right)}{r^{l} r^{\prime l^{\prime}}}\right|_{r, r^{\prime} \rightarrow 0}\right]=0$.

Under the same assumption of the zero-range approximation given in Eq. (16), the potential strength $V_{l}$ is related to the infinite volume two-body scattering phase shift $\delta_{l}\left(k_{\varepsilon}\right)$ by

$$
\frac{(4 \pi)^{2}}{2 \mu V_{l}}+\left.\frac{2^{2 l+1} \Gamma\left(l+\frac{1}{2}\right) \Gamma\left(l+\frac{3}{2}\right)}{\pi r^{2 l+1}}\right|_{r \rightarrow 0}=-k_{\varepsilon}^{2 l+1} \cot \delta_{l}\left(k_{\varepsilon}\right),
$$

see detailed discussions in Appendix B. The relative momentum $k_{\varepsilon}$ in infinite volume is related to the relative finite volume energy $\varepsilon$ by

$$
\frac{k_{\varepsilon}^{2}}{2 \mu}=\varepsilon+\triangle E_{R}, \quad \triangle E_{R}=\frac{Q B}{M}\left(n+\frac{1}{2}\right)-\frac{P_{x}^{2}+P_{y}^{2}}{2 M},
$$

where $\triangle E_{R}$ is the result of quantization of center-of-mass (CM) motion in a uniform magnetic field.

Eliminating $V_{l}$, Eqs. (18) and (19) together yield a Lüscher formulalike simple relation,

$$
\operatorname{det}\left[\delta_{l m, l^{\prime} m^{\prime}} \cot \delta_{l}\left(k_{\varepsilon}\right)-\mathcal{M}_{l m, l^{\prime} m^{\prime}}^{(B, L)}(\varepsilon)\right]=0
$$

where

$$
\begin{aligned}
\mathcal{M}_{l m, l^{\prime} m^{\prime}}^{(B, L)}(\varepsilon)= & -\left.\frac{2^{2 l^{\prime}+3} \Gamma^{2}\left(l^{\prime}+\frac{3}{2}\right)}{2 \mu k_{\varepsilon}^{2 l+1}(2 \pi)} \frac{G_{l m, l^{\prime} m^{\prime}}^{(B, L)}\left(r, r^{\prime} ; \varepsilon\right)}{r^{l} r^{\prime l^{\prime}}}\right|_{r, r^{\prime} \rightarrow 0} \\
& -\left.\delta_{l m, l^{\prime} m^{\prime}} \frac{2^{2 l+1} \Gamma\left(l+\frac{1}{2}\right) \Gamma\left(l+\frac{3}{2}\right)}{\pi} \frac{1}{\left(k_{\varepsilon} r\right)^{2 l+1}}\right|_{r \rightarrow 0} .
\end{aligned}
$$

The second term in $\mathcal{M}_{l m, l^{\prime} m^{\prime}}^{(B, L)}$ plays the role of the regulator of ultraviolet (UV) divergence and will cancel out the UV divergence in the finite volume magnetic Green's function, so that $\mathcal{M}_{l m, l^{\prime} m^{\prime}}^{(B, L)}$ is ultimately free of UV divergence. In general, the regularization and isolation of UV divergence in higher partial waves of a finite volume magnetic Green's function is a highly nontrivial task. Fortunately, it can be accomplished rather neatly for $S$-wave, hence, only the $S$-wave contribution will be considered in Sec. III. The regularization of UV divergence will be worked out explicitly.

\section{III. $S$-WAVE CONTRIBUTION AND CONTACT INTERACTION}

As was already mentioned in the previous section, the angular momentum basis in general is not a convenient basis for the dynamics of charged particles in a uniform magnetic field. The partial wave expansion of the finite volume magnetic Green's function and ultraviolet regularization can be tedious in general. Fortunately, if only the $S$-wave contribution is dominant, the formalism can be worked out nicely. In this section, only a contact interaction potential

$$
V(\mathbf{r})=\frac{V_{0}}{4 \pi} \delta(\mathbf{r})
$$

is used, which may be considered as the leading order contribution of chiral effective field theory and may be suitable for the few-body system, such as $\pi^{+}$interactions in finite volume.

With a contact interaction, the finite volume quantization condition is simply given by

$$
\frac{4 \pi}{V_{0}}=G_{B}^{(L)}(\mathbf{0}, \mathbf{0} ; \varepsilon) .
$$

In infinite volume, $V_{0}$ is related to the $S$-wave scattering amplitude by

$$
\frac{4 \pi}{V_{0}}-G^{(\infty)}\left(\mathbf{0} ; k_{\varepsilon}\right)=-\frac{2 \mu k_{\varepsilon}}{4 \pi} \frac{1}{t_{0}\left(k_{\varepsilon}\right)},
$$

where

$$
t_{0}\left(k_{\varepsilon}\right)=\frac{1}{\cot \delta_{0}\left(k_{\varepsilon}\right)-i},
$$

and infinite volume Green's function $G^{(\infty)}\left(\mathbf{0} ; k_{\varepsilon}\right)$ is given by

$G^{(\infty)}\left(\mathbf{0} ; k_{\varepsilon}\right)=\int \frac{d \mathbf{p}}{(2 \pi)^{3}} \frac{1}{\frac{k_{\varepsilon}^{2}}{2 \mu}-\frac{\mathbf{p}^{2}}{2 \mu}}=-i \frac{2 \mu k_{\varepsilon}}{4 \pi}-\left.\frac{2 \mu}{4 \pi r}\right|_{r \rightarrow 0}$.

Thus, the quantization condition is simply given by

$$
\cot \delta_{0}\left(k_{\varepsilon}\right)=-\frac{4 \pi}{2 \mu k_{\varepsilon}} G_{B}^{(L)}(\mathbf{0}, \mathbf{0} ; \varepsilon)-\left.\frac{1}{k_{\varepsilon} r}\right|_{r \rightarrow 0} .
$$

The magnetic Green's function $G_{B}^{(L)}(\mathbf{0}, \mathbf{0} ; \varepsilon)$ is a real function of $\varepsilon$. The UV divergent term

$$
-\left.\frac{1}{k_{\varepsilon} r}\right|_{r \rightarrow 0}
$$

plays the role of the UV counterterm that cancels out the UV divergent term in $G_{B}^{(L)}(\mathbf{0}, \mathbf{0} ; \varepsilon)$, so the ultimate result is finite and real as a function of $\varepsilon$. 


\section{A. Regularization of UV divergence}

In this section, we show explicitly how the UV divergence in $G_{B}^{(L)}(\mathbf{0}, \mathbf{0} ; \varepsilon)$ is regularized and isolated out explicitly. The UV divergence only appears when

$$
\mathbf{r}=(\boldsymbol{\rho}, 0) \rightarrow \mathbf{0},
$$

hence, a small $r=\rho$ is used as UV regulator. In the end, a final expression is obtained by taking the limit of $r=\rho \rightarrow 0$. Starting with an explicit expression of magnetic Green's function in CM frame $\left(\mathbf{P}_{B}=\mathbf{0}\right)$,

$$
\begin{aligned}
G_{B}^{(L)}(\mathbf{0}, \mathbf{0} ; \varepsilon)= & \sum_{n_{x}, n_{y} \in \mathbb{Z}} e^{-\frac{i q B}{2} n_{x} n_{q} L n_{y} L} \\
& \times e^{-\frac{q B}{4}\left|\boldsymbol{\rho}+n_{x} n_{q} L \mathbf{e}_{x}+n_{y} L \mathbf{e}_{y}\right|^{2}} \frac{q B}{2 \pi} \frac{1}{L} \sum_{k_{z}=\frac{2 \pi n_{z}}{L}}^{n_{z} \in \mathbb{Z}} \\
& \times\left.\sum_{n=0}^{\infty} \frac{L_{n}\left(\frac{q B}{2}\left|\boldsymbol{\rho}+n_{x} n_{q} L \mathbf{e}_{x}+n_{y} L \mathbf{e}_{y}\right|^{2}\right)}{\varepsilon-\frac{q B}{\mu}\left(n+\frac{1}{2}\right)-\frac{k_{z}^{2}}{2 \mu}}\right|_{\boldsymbol{\rho} \rightarrow 0} .
\end{aligned}
$$

The UV divergence is associated to the term

$\frac{1}{L} \sum_{k_{z}=\frac{2 \pi n_{z}}{L}}^{n_{z} \in \mathbb{Z}} \sum_{n=0}^{\infty} \frac{1}{\varepsilon-\frac{q B}{\mu}\left(n+\frac{1}{2}\right)-\frac{k_{z}^{2}}{2 \mu}} \propto \int^{\Lambda=\frac{1}{r}} \frac{d k^{3}}{k^{2}} \propto \Lambda=\left.\frac{1}{r}\right|_{r \rightarrow 0}$,

hence $G_{B}^{(L)}$ is linearly divergent.

The linear divergence can be regularized simply by subtraction. Therefore, we first split the finite volume magnetic Green's function into a regularized term by subtraction and a term that is UV divergent,

$$
G_{B}^{(L)}(\mathbf{0}, \mathbf{0} ; \varepsilon)=\triangle G_{B}^{(L)}(\varepsilon)+G_{B}^{(L)}(\mathbf{0}, \mathbf{0} ; 0),
$$

where

$$
\triangle G_{B}^{(L)}(\varepsilon)=G_{B}^{(L)}(\mathbf{0}, \mathbf{0} ; \varepsilon)-G_{B}^{(L)}(\mathbf{0}, \mathbf{0} ; 0) .
$$

The subtracted term $\triangle G_{B}^{(L)}(\varepsilon)$ is free of UV divergence. Using the identity

$$
\frac{1}{L} \sum_{k_{z}=\frac{2 \pi n_{z}}{L}}^{n_{z} \in \mathbb{Z}} \frac{1}{E-\frac{k_{z}^{2}}{2 \mu}}=\frac{2 \mu}{2 \sqrt{2 \mu E}} \cot \frac{\sqrt{2 \mu E} L}{2},
$$

$\triangle G_{B}^{(L)}(\varepsilon)$ is thus given explicitly by

$$
\begin{aligned}
& \triangle G_{B}^{(L)}(\varepsilon)=\frac{2 \mu q B}{4 \pi} \sum_{n=0}^{\infty} \sum_{n_{x}, n_{y} \in \mathbb{Z}} e^{-\frac{i q B n_{x} n_{y} \eta_{q} L^{2}}{2}} \\
& \times e^{-\frac{q B}{4}\left|n_{x} n_{q} L \mathbf{e}_{x}+n_{y} L \mathbf{e}_{y}\right|^{2}} L_{n}\left(\frac{q B}{2}\left|n_{x} n_{q} L \mathbf{e}_{x}+n_{y} L \mathbf{e}_{y}\right|^{2}\right) \\
& \times\left[\frac{\cot \frac{\sqrt{2 \mu \varepsilon-2 q B\left(n+\frac{1}{2}\right)} L}{2}}{\sqrt{2 \mu \varepsilon-2 q B\left(n+\frac{1}{2}\right)}}+\frac{\operatorname{coth} \frac{\sqrt{2 q B\left(n+\frac{1}{2}\right)}}{2}}{\sqrt{2 q B\left(n+\frac{1}{2}\right)}}\right] \text {. }
\end{aligned}
$$

Next, the UV divergence in $G_{B}^{(L)}(\mathbf{0}, \mathbf{0} ; 0)$ can be isolated out by further splitting $G_{B}^{(L)}(\mathbf{0}, \mathbf{0} ; 0)$ into

$$
G_{B}^{(L)}(\mathbf{0 ,} \mathbf{0} ; 0)=G_{\mathrm{UV}}^{(B, L)}+G_{R C}^{(B, L)},
$$

where $G_{\mathrm{UV}}^{(B, L)}$ is UV divergent and is given by

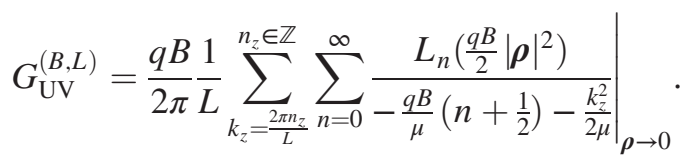

The $G_{R C}^{(B, L)}$ is a regulated constant term, and is defined by

$$
\begin{aligned}
G_{R C}^{(B, L)} & =\sum_{n_{x}, n_{y} \neq 0} e^{-\frac{i q B n_{x} n_{y} n_{q} L^{2}}{2}} \frac{q B}{2 \pi} \frac{1}{L} \sum_{k_{z}=\frac{2 \pi n_{z}}{L}}^{n_{z} \in \mathbb{Z}} \\
& \times \sum_{n=0}^{\infty} \frac{e^{-\frac{q B}{4}\left|n_{x} n_{q} L \mathbf{e}_{x}+n_{y} L \mathbf{e}_{y}\right|^{2} L_{n}\left(\frac{q B}{2}\left|n_{x} n_{q} L \mathbf{e}_{x}+n_{y} L \mathbf{e}_{y}\right|^{2}\right)}}{-\frac{q B}{\mu}\left(n+\frac{1}{2}\right)-\frac{k_{z}^{2}}{2 \mu}} .
\end{aligned}
$$

(1) The regulated constant term $G_{R C}^{(B, L)}$ can be further simplified by using the identity

$$
\begin{aligned}
& \sum_{n=0}^{\infty} \frac{L_{n}\left(\frac{q B}{2} x^{2}\right)}{-\frac{q B}{\mu}\left(n+\frac{1}{2}\right)-\frac{k_{z}^{2}}{2 \mu}} \\
& \quad=-\frac{\mu}{q B} \Gamma\left(\frac{1}{2}+\frac{k_{z}^{2}}{2 q B}\right) U\left(\frac{1}{2}+\frac{k_{z}^{2}}{2 q B}, 1, \frac{q B}{2} x^{2}\right)
\end{aligned}
$$

where $U(a, b, z)$ stands for the Kummer function, hence, we find 


$$
\begin{aligned}
G_{R C}^{(B, L)}= & -\frac{2 \mu}{4 \pi} \frac{1}{L} \sum_{k_{z}=\frac{2 \pi n_{z}}{L}}^{n_{z} \in \mathbb{Z}} \Gamma\left(\frac{1}{2}+\frac{k_{z}^{2}}{2 q B}\right) \\
& \times \sum_{n_{x}, n_{y} \neq 0} e^{-\frac{i q B n_{x} n_{y} n_{q} L^{2}}{2}} e^{-\frac{q B}{4}\left|n_{x} n_{q} L \mathbf{e}_{x}+n_{y} L \mathbf{e}_{y}\right|^{2}} \\
& \times U\left(\frac{1}{2}+\frac{k_{z}^{2}}{2 q B}, 1, \frac{q B}{2}\left|n_{x} n_{q} L \mathbf{e}_{x}+n_{y} L \mathbf{e}_{y}\right|^{2}\right) .
\end{aligned}
$$

Asymptotically, the Kummer function decays exponentially,

$$
\begin{aligned}
& e^{-\frac{q B}{4} x^{2}} \Gamma\left(\frac{1}{2}+\frac{k_{z}^{2}}{2 q B}\right) U\left(\frac{1}{2}+\frac{k_{z}^{2}}{2 q B}, 1, \frac{q B}{2} x^{2}\right) \\
& \stackrel{k_{z} \rightarrow \infty}{\rightarrow} 2 K_{0}\left(\sqrt{k_{z}^{2} x^{2}}\right),
\end{aligned}
$$

hence $G_{R C}^{(B, L)}$ is indeed a well-defined regulated constant.

(2) The explicit expression of the UV divergence in $G_{\mathrm{UV}}^{(B, L)}$ can be worked out. First of all, the infinite sum of integer $n \in[0, \infty]$ in Eq. (35) is split into $n \in\left[0, n_{\Lambda}\right]$ and $n \in\left[n_{\Lambda}, \infty\right]$, where $n_{\Lambda}$ serves as a cutoff integer and $n_{\Lambda} \gg 1$. For large $n_{\Lambda}$, the summation is replaced by integration, hence one can rewrite Eq. (35) to

$$
\begin{aligned}
G_{\mathrm{UV}}^{(B, L)}= & \frac{q B}{2 \pi} \frac{1}{L} \sum_{k_{z}=\frac{2 \pi n_{z}}{L}}^{n_{z} \in \mathbb{Z}} \sum_{n=0}^{n_{\Lambda}} \frac{L_{n}\left(\frac{q B}{2}|\rho|^{2}\right)}{-\frac{q B}{\mu}\left(n+\frac{1}{2}\right)-\left.\frac{k_{z}^{2}}{2 \mu}\right|_{\rho \rightarrow 0}} \\
& +\left.\frac{q B}{2 \pi} \frac{1}{L} \sum_{k_{z}=\frac{2 \pi n_{z}}{L}}^{n_{z} \in \mathbb{Z}} \int_{n_{\Lambda}}^{\infty} d n \frac{L_{n}\left(\frac{q B}{2}|\rho|^{2}\right)}{-\frac{q B}{\mu}\left(n+\frac{1}{2}\right)-\frac{k_{z}^{2}}{2 \mu}}\right|_{\rho \rightarrow 0} .
\end{aligned}
$$

Let us rewrite it further to

$$
\begin{aligned}
G_{\mathrm{UV}}^{(B, L)}= & \left.\frac{q B}{2 \pi} \frac{1}{L} \sum_{k_{z}=\frac{2 \pi n_{z}}{L}}^{n_{z} \in \mathbb{Z}}\left[\sum_{n=0}^{n_{\Lambda}}-\int_{0}^{n_{\Lambda}} d n\right] \frac{L_{n}\left(\frac{q B}{2}|\rho|^{2}\right)}{-\frac{q B}{\mu}\left(n+\frac{1}{2}\right)-\frac{k_{z}^{2}}{2 \mu}}\right|_{\rho \rightarrow 0} \\
& +\left.\frac{q B}{2 \pi} \frac{1}{L} \sum_{k_{z}=\frac{2 \pi n_{z}}{L}}^{n_{z} \in \mathbb{Z}} \int_{0}^{\infty} d n \frac{L_{n}\left(\frac{q B}{2}|\rho|^{2}\right)}{-\frac{q B}{\mu}\left(n+\frac{1}{2}\right)-\frac{k_{z}^{2}}{2 \mu}}\right|_{\rho \rightarrow 0} .
\end{aligned}
$$

The first term in Eq. (41) is finite, so $\rho$ can be set to zero safely. In the second term in Eq. (41), let us rescale the integral dummy variable $d n$ to

$$
q B \rho^{2} d n \rightarrow \xi d \xi, \quad q B \rho^{2} n \rightarrow \frac{\xi^{2}}{2}
$$

thus, the Eq. (35) is then turned into

$$
\begin{aligned}
G_{\mathrm{UV}}^{(B, L)}= & \frac{q B}{2 \pi} \frac{1}{L} \sum_{k_{z}=\frac{2 \pi n_{z}}{L}}^{n_{z} \in \mathbb{Z}}\left[\sum_{n=0}^{n_{\Lambda}}-\int_{0}^{n_{\Lambda}} d n\right] \frac{1}{-\frac{q B}{\mu}\left(n+\frac{1}{2}\right)-\frac{k_{z}^{2}}{2 \mu}} \\
& +\left.\frac{1}{2 \pi} \frac{1}{L} \sum_{k_{z}=\frac{2 \pi n_{z}}{L}}^{n_{z} \in \mathbb{Z}} \int_{0}^{\infty} \xi d \xi \frac{L_{\frac{\xi^{2}}{2 q B r^{2}}}\left(\frac{q B r^{2}}{2}\right)}{-\frac{\xi^{2}}{2 \mu}-\frac{q B r^{2}}{2 \mu}-\frac{k_{z}^{2} r^{2}}{2 \mu}}\right|_{r \rightarrow 0} .
\end{aligned}
$$

Using the asymptotic form of the Laguerre polynomial,

$$
L_{\frac{\xi^{2}}{2 q B r^{2}}}\left(\frac{q B r^{2}}{2}\right) \stackrel{r \rightarrow 0}{\rightarrow} J_{0}\left(\sqrt{\xi^{2}}\right)
$$

and identity in Eq. (32) again, we find

$G_{\mathrm{UV}}^{(B, L)}=\triangle G_{\mathrm{UV}}^{(B, L)}$

$$
-\left.\frac{2 \mu}{2 \pi r} \int_{0}^{\infty} \xi d \xi J_{0}\left(\sqrt{\xi^{2}}\right) \frac{\operatorname{coth} \frac{\sqrt{\frac{\xi^{2}}{r^{2}}+q B L}}{2}}{2 \sqrt{\xi^{2}+q B r^{2}}}\right|_{r \rightarrow 0},
$$

where

$$
\triangle G_{\mathrm{UV}}^{(B, L)}=-\frac{2 \mu q B}{2 \pi}\left[\sum_{n=0}^{n_{\Lambda}}-\int_{0}^{n_{\Lambda}} d n\right] \frac{\operatorname{coth} \frac{\sqrt{2 q B\left(n+\frac{1}{2}\right)} L}{2}}{2 \sqrt{2 q B\left(n+\frac{1}{2}\right)}} .
$$

As $r \rightarrow 0$ and $\frac{1}{r} \rightarrow \infty$,

$\operatorname{coth} z \stackrel{z \rightarrow \infty}{\sim} 1+2 e^{-2 z}+2 e^{-4 z} \cdots, \quad z=\frac{\sqrt{\frac{\xi^{2}}{r^{2}}+q B L}}{2}$,

and also using identity

$$
\int_{0}^{\infty} \xi d \xi \frac{J_{0}\left(\sqrt{\xi^{2}}\right)}{2 \sqrt{\xi^{2}+q B r^{2}}}=\frac{2 \pi}{4 \pi} e^{-\sqrt{q B} r}
$$

we finally obtain a explicit expression of UV divergence,

$$
\begin{aligned}
G_{\mathrm{UV}}^{(B, L)} & =\Delta G_{\mathrm{UV}}^{(B, L)}-\left.\frac{2 \mu e^{-\sqrt{q B} r}}{4 \pi r}\right|_{r \rightarrow 0} \\
& =\Delta G_{\mathrm{UV}}^{(B, L)}+\frac{2 \mu \sqrt{q B}}{4 \pi}-\left.\frac{2 \mu}{4 \pi r}\right|_{r \rightarrow 0} .
\end{aligned}
$$


Putting all the pieces together, we thus find

$$
\begin{aligned}
& G_{B}^{(L)}(\mathbf{0}, \mathbf{0} ; \varepsilon) \\
& \quad=\Delta G_{B}^{(L)}(\varepsilon)+G_{R C}^{(B, L)}+\triangle G_{\mathrm{UV}}^{(B, L)}+\frac{2 \mu \sqrt{q B}}{4 \pi}-\left.\frac{2 \mu}{4 \pi r}\right|_{r \rightarrow 0},
\end{aligned}
$$

where $\triangle G_{B}^{(L)}(\varepsilon), G_{R C}^{(B, L)}$, and $\triangle G_{\mathrm{UV}}^{(B, L)}$ are all free of UV divergence and given by Eq. (33), Eq. (38), and Eq. (46) respectively.

\section{B. Regulated $S$-wave quantization condition}

With explicitly isolated UV divergence in the finite volume magnetic Green's function in Eq. (50), the UV divergent terms in quantization condition given by Eq. (27) cancel out, thus we find a regulated quantization condition

$$
\cot \delta_{0}\left(k_{\varepsilon}\right)=\mathcal{M}_{0,0}^{(B, L)}(\varepsilon),
$$

where

$$
\begin{aligned}
& \mathcal{M}_{0,0}^{(B, L)}(\varepsilon) \\
& =-\frac{4 \pi}{2 \mu k_{\varepsilon}}\left[\triangle G_{B}^{(L)}(\varepsilon)+G_{R C}^{(B, L)}+\triangle G_{\mathrm{UV}}^{(B, L)}\right]-\frac{\sqrt{q B}}{k_{\varepsilon}} .
\end{aligned}
$$

The expression of $\triangle G_{B}^{(L)}(\varepsilon), G_{R C}^{(B, L)}$, and $\triangle G_{\mathrm{UV}}^{(B, L)}$ are given by Eq. (33), Eq. (38), and Eq. (46) respectively.

\section{Lüscher formula at the limit of $q B \rightarrow 0$}

Using the identity given in Eq. (37), Eq. (28) can be rewritten as

$$
\begin{aligned}
G_{B}^{(L)}(\mathbf{0 , 0} ; \varepsilon)= & -\frac{2 \mu}{4 \pi} \frac{1}{L} \sum_{k_{z}=\frac{2 \pi n_{z}}{L}}^{n_{z} \in \mathbb{Z}} \Gamma\left(\frac{1}{2}+\frac{k_{z}^{2}-2 \mu \varepsilon}{2 q B}\right) \\
& \times \sum_{n_{x}, n_{y}} e^{-\frac{q q B n_{x} n_{y} n_{q} L^{2}}{2}} e^{-\frac{q B}{4}\left|n_{x} n_{q} L \mathbf{e}_{x}+n_{y} L \mathbf{e}_{y}\right|^{2}} \\
& \times U\left(\frac{1}{2}+\frac{k_{z}^{2}-2 \mu \varepsilon}{2 q B}, 1, \frac{q B}{2}\left|n_{x} n_{q} L \mathbf{e}_{x}+n_{y} L \mathbf{e}_{y}\right|^{2}\right) .
\end{aligned}
$$

At the limit of $q B \rightarrow 0$, using the asymptotic form of

$$
\begin{gathered}
\Gamma\left(\frac{1}{2}+\frac{k_{z}^{2}-2 \mu \varepsilon}{2 q B}\right) U\left(\frac{1}{2}+\frac{k_{z}^{2}-2 \mu \varepsilon}{2 q B}, 1, \frac{q B}{2} r^{2}\right) \\
\stackrel{q B \rightarrow 0}{\rightarrow} i \pi H_{0}^{(1)}\left(\sqrt{2 \mu \varepsilon-k_{z}^{2}} r\right)+\mathcal{O}(q B),
\end{gathered}
$$

and also taking $n_{q}=1$, thus one finds

$$
\begin{aligned}
& G_{B}^{(L)}(\mathbf{0}, \mathbf{0} ; \varepsilon) \stackrel{q B \rightarrow 0}{\rightarrow}-\frac{2 \mu}{L} \sum_{k_{z}=\frac{2 \pi n_{z}}{L}}^{n_{z} \in \mathbb{Z}} \\
& \quad \times \sum_{n_{x}, n_{y}} \frac{i}{4} H_{0}^{(1)}\left(\sqrt{2 \mu \varepsilon-k_{z}^{2}}\left|n_{x} L \mathbf{e}_{x}+n_{y} L \mathbf{e}_{y}\right|\right)+\mathcal{O}(q B) .
\end{aligned}
$$

Next, using the identity

$$
\begin{gathered}
-\sum_{n_{x}, n_{y}} \frac{i}{4} H_{0}^{(1)}\left(\sqrt{2 \mu \varepsilon-k_{z}^{2}}\left|n_{x} L \mathbf{e}_{x}+n_{y} L \mathbf{e}_{y}\right|\right) \\
=\frac{1}{L^{2}} \sum_{k_{x, y}=\frac{2 \pi n_{x, y}}{L}, n_{x, y} \in \mathbb{Z}} \frac{1}{2 \mu \varepsilon-k_{z}^{2}-k_{x}^{2}-k_{y}^{2}},
\end{gathered}
$$

one thus can easily show that

$$
G_{B}^{(L)}(\mathbf{0}, \mathbf{0} ; \varepsilon) \stackrel{q B \rightarrow 0}{\rightarrow} G_{0}^{(L)}\left(\mathbf{0}, k_{\varepsilon}\right)+\mathcal{O}(q B),
$$

where

$$
G_{0}^{(L)}(\mathbf{r}, k)=\frac{2 \mu}{L^{3}} \sum_{\mathbf{p}=\frac{2 \pi}{L} \mathbf{n}, \mathbf{n} \in \mathbb{Z}^{3}} \frac{e^{i \mathbf{p} \cdot \mathbf{r}}}{k^{2}-\mathbf{p}^{2}} .
$$

Hence, the finite volume magnetic zeta function $\mathcal{M}_{0,0}^{(B, L)}(\varepsilon)$ at the limit of $q B \rightarrow 0$ is given by

$$
\mathcal{M}_{0,0}^{(B, L)}(\varepsilon) \stackrel{q B \rightarrow 0}{\rightarrow} \mathcal{M}_{0,0}^{(L)}\left(k_{\varepsilon}\right)+\mathcal{O}(q B),
$$

which is consistent with the perturbation result given in Ref. [71]. The $\mathcal{M}_{0,0}^{(L)}\left(k_{\varepsilon}\right)$ denotes the regular finite volume zeta function, see $[1,2,8]$, and is defined by

$$
\mathcal{M}_{0,0}^{(L)}\left(k_{\varepsilon}\right)=-\frac{4 \pi}{k_{\varepsilon} L^{3}} \sum_{\mathbf{p}=\frac{2 \pi}{L} \mathbf{n}, \mathbf{n} \in \mathbb{Z}^{3}} \frac{e^{\frac{k_{\varepsilon}^{2}-\mathbf{p}^{2}}{\Lambda^{2}}}}{k_{\varepsilon}^{2}-\mathbf{p}^{2}}+\frac{\Lambda}{k_{\varepsilon} \sqrt{\pi}}\left[\sum_{\mathbf{n} \in \mathbb{Z}^{3}}^{\mathbf{n} \neq 0} \int_{1}^{\infty} d \xi e^{-\frac{(\mathbf{n} L \Lambda \xi)^{2}}{4}+\frac{k^{2}}{(\Lambda \xi)^{2}}}+\sum_{n=0}^{\infty} \frac{\left(\frac{k_{\varepsilon}^{2}}{\Lambda^{2}}\right)^{n}}{n !(2 n-1)}\right]
$$

where $\Lambda$ is an arbitrary UV regulator.

The comparison of the finite volume magnetic zeta function $\mathcal{M}_{0,0}^{(B, L)}(\varepsilon)$ and regular finite volume zeta function $\mathcal{M}_{0,0}^{(L)}(k)$ are shown in Fig. 1. The splitting of energy levels is illustrated in the comparison of the curves of $\mathcal{M}_{0,0}^{(B, L)}(\varepsilon)$ in the upper and lower panels with $n_{q}=1$ and $n_{q}=2$ respectively; the number of $\mathcal{M}_{0,0}^{(B, L)}(\varepsilon)$ curves double as the value of $n_{q}$ is doubled. 

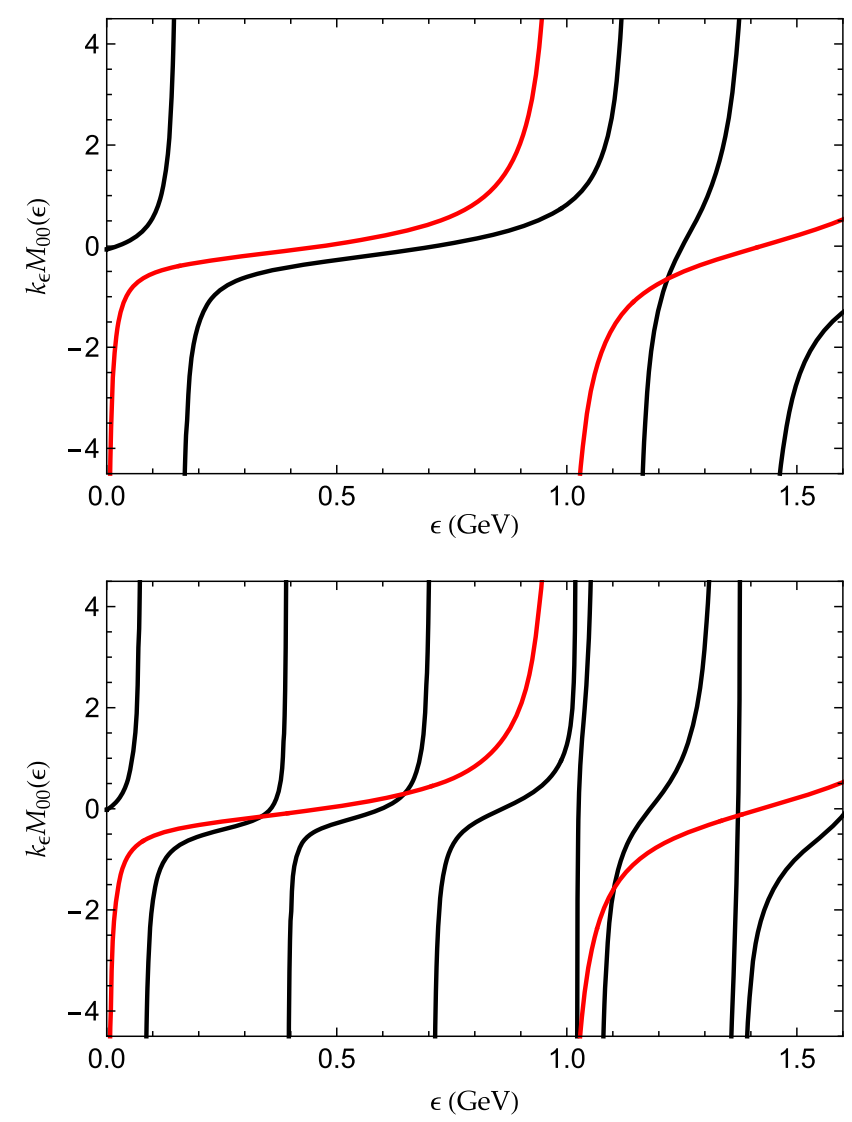

FIG. 1. Comparison of the finite volume magnetic zeta function $k_{\varepsilon} \mathcal{M}_{0,0}^{(B, L)}(\varepsilon)$ (black solid) and regular finite volume zeta function $k \mathcal{M}_{0,0}^{(L)}(k)$ (red solid) in Eqs. (52) and (60) respectively. The parameters are chosen as $\mu=0.2 \mathrm{GeV}, L=10 \mathrm{GeV}^{-1}, n_{p}=1$, and $n_{q}=1,2$ in the upper and lower panels respectively.

\section{SUMMARY}

A formalism for describing charged spinless bosons interaction in both a finite volume and a magnetic field is presented in this work. We show that for a short-range potential, a Lüscher formulalike relation that relates discrete energy spectrum to scattering phase shifts can be obtained. The regularization of UV divergence is worked out explicitly for $S$-wave contribution; the regulated $S$-wave quantization condition may be useful for the LQCD study of charged boson system, such as a $\pi^{+}$or $K^{+}$system. In finite volume and in a magnetic field, translation symmetry of a system is only preserved when the magnetic flux, $\Phi_{B}=q B L^{2}$, is given by $2 \pi$ multiplied by a rational number $n_{p} / n_{q}$ where $n_{p}$ and $n_{q}$ are relatively prime integers. The presence of a magnetic field thus results in the splitting of the energy level into $n_{q}$ subenergy levels.

\section{ACKNOWLEDGMENTS}

P. G. acknowledges support from the Department of Physics and Engineering, California State University,
Bakersfield, CA. This research (P. G.) was also supported in part by the U.S. National Science Foundation under Grant No. NSF PHY-1748958.

\section{APPENDIX A: TWO CHARGED BOSONS IN A UNIFORM MAGNETIC FIELD}

The dynamics of two charged nonrelativistic identical bosons in a uniform magnetic field is described by THE Schrödinger equation,

$$
\left[E+\sum_{i=1}^{2} \frac{\left(\nabla_{i}+i e \mathbf{A}\left(\mathbf{x}_{i}\right)\right)^{2}}{2 m}-V\left(\mathbf{x}_{1}-\mathbf{x}_{2}\right)\right] \Psi_{E}\left(\mathbf{x}_{1}, \mathbf{x}_{2}\right)=0
$$

where $m$ is the mass of identical bosons. $\mathbf{x}_{i}$ denotes the position of the $i$ th particle, and the short-range interaction between two particles is represented by $V\left(\mathbf{x}_{1}-\mathbf{x}_{2}\right)$. $\mathbf{A}\left(\mathbf{x}_{i}\right)$ stands for the vector potential of a uniform magnetic field.

Throughout the entire work, the uniform magnetic field is assumed along the $z$ axis, $\mathbf{B}=B \mathbf{e}_{z}$, and the vector potential in Landau gauge is used,

$$
\mathbf{A}\left(\mathbf{x}_{i}\right)=B\left(0, x_{i}, 0\right) .
$$

The solutions of the Schrödinger equation in other gauges are obtained by a gauge transformation through a scalar field, $\chi\left(\mathbf{x}_{i}\right)$,

$$
\mathbf{A}\left(\mathbf{x}_{i}\right) \rightarrow \mathbf{A}\left(\mathbf{x}_{i}\right)-\nabla \chi\left(\mathbf{x}_{i}\right)
$$

and

$$
\Psi_{E}\left(\mathbf{x}_{1}, \mathbf{x}_{2}\right) \rightarrow e^{i e \sum_{i=1}^{2} \chi\left(\mathbf{x}_{i}\right)} \Psi_{E}\left(\mathbf{x}_{1}, \mathbf{x}_{2}\right) .
$$

\section{Separation of center of mass and relative motions}

The center-of-mass motion (CM) and relative motion of two particles can be separated by introducing $\mathrm{CM}$ and relative coordinates respectively

$$
\mathbf{R}=\frac{\mathbf{x}_{1}+\mathbf{x}_{2}}{2}, \quad \mathbf{r}=\mathbf{x}_{1}-\mathbf{x}_{2} .
$$

Therefore, the Hamiltonian has a separable form and the total two particles wave function is given by the product of $\mathrm{CM}$ and relative wave functions,

$$
\Psi_{E}\left(\mathbf{x}_{1}, \mathbf{x}_{2}\right)=\Phi_{E-\varepsilon}(\mathbf{R}) \psi_{\varepsilon}(\mathbf{r}),
$$

where the CM wave function, $\Phi_{E-\varepsilon}(\mathbf{R})$, and relative wave function, $\psi_{\varepsilon}(\mathbf{r})$, satisfy Schrödinger equations respectively, 


$$
\left[(E-\varepsilon)+\frac{\left(\nabla_{\mathbf{R}}+i Q \mathbf{A}(\mathbf{R})\right)^{2}}{2 M}\right] \Phi_{E-\varepsilon}(\mathbf{R})=0
$$

and

$$
\left[\varepsilon+\frac{\left(\nabla_{\mathbf{r}}+i q \mathbf{A}(\mathbf{r})\right)^{2}}{2 \mu}-V(\mathbf{r})\right] \psi_{\varepsilon}(\mathbf{r})=0 .
$$

The total and reduced mass of two particles are

$$
M=2 m \quad \text { and } \quad \mu=\frac{m}{2}
$$

respectively, and similarly

$$
Q=2 e \quad \text { and } \quad q=\frac{e}{2}
$$

are total and reduced charges respectively.

\section{Magnetic translation group and magnetic periodic boundary condition}

Now, let us consider putting charged particles in a periodic cubic box with size $L$, and interaction between two particles is also periodic,

$$
V(\mathbf{r}+\mathbf{n} L)=V(\mathbf{r}), \quad \mathbf{n} \in \mathbb{Z}^{3} .
$$

Without a magnetic field, the discrete translation symmetry of a system in finite volume yields the conserved total momentum of a system with discrete values:

$$
\mathbf{P}=\frac{2 \pi \mathbf{n}}{L}, \quad \mathbf{n} \in \mathbb{Z}^{3} .
$$

In a magnetic field, though the potential $V$ is periodic, the $\hat{H}_{\mathbf{r}}$ is not discrete translation invariant

$$
\hat{H}_{\mathbf{r}+\mathbf{n} L} \neq \hat{H}_{\mathbf{r}}
$$

due to the fact that the vector potential is coordinate dependent and breaks discrete translation symmetry

$$
\mathbf{A}\left(\mathbf{r}+n L \mathbf{e}_{x}\right)=\mathbf{A}(\mathbf{r})+B n L \mathbf{e}_{y}, \quad n \in \mathbb{Z} .
$$

The momentum operator, $\hat{\mathbf{p}}=-i \nabla_{\mathbf{r}}$, does not commute with $\hat{H}_{\mathbf{r}}$ :

$$
\left[\hat{\mathbf{p}}, \hat{H}_{\mathbf{r}}\right] \neq 0 .
$$

Hence canonical momentum is no longer a conserved quantity as the consequence of breaking down of discrete translation symmetry in a uniform magnetic field. It has been shown in Refs. [75-77] that a pseudomomentum operator
$\hat{\mathbf{K}}_{\mathbf{r}}=-i \nabla_{\mathbf{r}}+q \mathbf{A}(\mathbf{r})-q \mathbf{B} \times \mathbf{r}=-i \nabla_{\mathbf{r}}+q B\left(r_{y}, 0,0\right)$

in fact commutes with $\hat{H}_{\mathbf{r}}$ :

$$
\left[\hat{\mathbf{K}}_{\mathbf{r}}, \hat{H}_{\mathbf{r}}\right]=0 .
$$

Therefore, $\hat{\mathbf{K}}_{\mathbf{r}}$ can be used as generator of a magnetic translation operator,

$$
\hat{T}_{\mathbf{r}}(\mathbf{n} L)=e^{i \hat{\mathbf{K}}_{\mathbf{r}} \cdot \mathbf{n} L}=e^{i\left(-i \nabla_{\mathbf{r}}+q \mathbf{A}(\mathbf{r})-q \mathbf{B} \times \mathbf{r}\right) \cdot \mathbf{n} L},
$$

where $e^{i\left(-i \nabla_{\mathbf{r}}\right) \cdot \mathbf{n} L}$ is the pure translation operator, and

$$
e^{i\left(-i \nabla_{\mathbf{r}}\right) \cdot \mathbf{n} L} \psi_{\varepsilon}(\mathbf{r})=\psi_{\varepsilon}(\mathbf{r}+\mathbf{n} L) .
$$

So that

$$
\hat{T}_{\mathbf{r}}(\mathbf{n} L) \psi_{\varepsilon}(\mathbf{r})=e^{i q(\mathbf{A}(\mathbf{r})-\mathbf{B} \times \mathbf{r}) \cdot \mathbf{n} L} \psi_{\varepsilon}(\mathbf{r}+\mathbf{n} L),
$$

and $\hat{T}_{\mathbf{r}}$ commutes with the Hamiltonian,

$$
\left[\hat{T}_{\mathbf{r}}, \hat{H}\right]=0,
$$

which leaves the Hamiltonian invariant. However, the magnetic translation operators do not commute with each other in general,

$\hat{T}_{\mathbf{r}}\left(n_{x} L \mathbf{e}_{x}\right) \hat{T}_{\mathbf{r}}\left(n_{y} L \mathbf{e}_{y}\right)=e^{-i q B L^{2} n_{x} n_{y}} \hat{T}_{\mathbf{r}}\left(n_{y} L \mathbf{e}_{y}\right) \hat{T}_{\mathbf{r}}\left(n_{x} L \mathbf{e}_{x}\right)$,

where $\left(n_{x}, n_{y}\right) \in \mathbb{Z}$.

As shown in Refs. [75,76], when the values of $q B$ are taken as

$$
q B=\frac{1}{2} e B=\frac{2 \pi}{L^{2}} \frac{n_{p}}{n_{q}}
$$

where $n_{p}$ and $n_{q}$ are integers that are relatively prime. The magnetic translation operators with an enlarged unit cell formed by the increased size of $n_{q} L$ in the $\mathbf{e}_{x}$ direction thus commute with each other,

$$
\left[\hat{T}_{\mathbf{r}}\left(n_{x}\left(n_{q} L\right) \mathbf{e}_{x}\right), \hat{T}_{\mathbf{r}}\left(n_{y} L \mathbf{e}_{y}\right)\right]=0 .
$$

Therefore magnetic translation operators with an enlarged magnetic unit box that is defined by

$$
n_{q} L \mathbf{e}_{x} \times L \mathbf{e}_{y} \times L \mathbf{e}_{z}
$$

form a discrete group that are commonly referred to as a magnetic translation group. 
The translation operator for two charged particles can be introduced by

$$
\hat{T}_{\mathbf{x}_{1}, \mathbf{x}_{2}}\left(\mathbf{n}_{1 B} L, \mathbf{n}_{2 B} L\right)=\hat{T}_{\mathbf{x}_{1}}\left(\mathbf{n}_{1 B} L\right) \hat{T}_{\mathbf{x}_{2}}\left(\mathbf{n}_{2 B} L\right)
$$

where

$$
\hat{T}_{\mathbf{x}_{i}}(\mathbf{n} L)=e^{i\left(-i \nabla_{\mathbf{x}_{i}}+e \mathbf{A}\left(\mathbf{x}_{i}\right)-e \mathbf{B} \times \mathbf{x}_{i}\right) \cdot \mathbf{n} L} .
$$

Both $\mathbf{n}_{1 B} L$ and $\mathbf{n}_{2 B} L$ are defined in an enlarged magnetic unit box,

$\mathbf{n}_{i B} L=n_{i x}\left(n_{q} L\right) \mathbf{e}_{x}+n_{i y} L \mathbf{e}_{y}+n_{i z} L \mathbf{e}_{z}, \quad n_{i x, i y, i z} \in \mathbb{Z}$.

We may rewrite two particles translation operator in terms of $\mathrm{CM}$ and relative motion quantities,

$\hat{T}_{\mathbf{x}_{1}, \mathbf{x}_{2}}\left(\mathbf{n}_{1 B} L, \mathbf{n}_{2 B} L\right)=\hat{T}_{\mathbf{r}}\left(\mathbf{n}_{1 B} L-\mathbf{n}_{2 B} L\right) \hat{T}_{\mathbf{R}}\left(\frac{\mathbf{n}_{1 B}+\mathbf{n}_{2 B}}{2} L\right)$

where $\hat{T}_{\mathbf{r}}$ is defined in Eq. (A12), and

$$
\hat{T}_{\mathbf{R}}\left(\frac{\mathbf{n}_{B} L}{2}\right)=e^{i\left(-i \nabla_{\mathbf{R}}+Q \mathbf{A}(\mathbf{R})-Q \mathbf{B} \times \mathbf{R}\right) \cdot \frac{\mathbf{n}_{B} L}{2}}
$$

Note that

$$
Q B=2 e B=\frac{2 \pi}{\left(\frac{L}{2}\right)^{2}} \frac{n_{p}}{n_{q}}
$$

and the translation operation of the CM motion may be considered as a motion of a composite charge particle with total charges of $Q=2 e$ in a periodic box with size $L / 2$.

The magnetic translation invariance of a system yields

$$
\hat{T}_{\mathbf{x}_{1}, \mathbf{x}_{2}}\left(\mathbf{n}_{1 B} L, \mathbf{n}_{2 B} L\right) \Psi_{E}\left(\mathbf{x}_{1}, \mathbf{x}_{2}\right)=\Psi_{E}\left(\mathbf{x}_{1}, \mathbf{x}_{2}\right) .
$$

Using Eq. (A19), the boundary conditions for two particles in both finite volume and a uniform magnetic field is given by

$$
\begin{aligned}
& \Psi_{E}\left(\mathbf{x}_{1}+\mathbf{n}_{1 B} L, \mathbf{x}_{2}+\mathbf{n}_{2 B} L\right) \\
& =e^{-i e \sum_{i=1}^{2}\left(\mathbf{A}\left(\mathbf{x}_{i}\right)-\mathbf{B} \times \mathbf{x}_{i}\right) \cdot \mathbf{n}_{i B} L} \Psi_{E}\left(\mathbf{x}_{1}, \mathbf{x}_{2}\right) .
\end{aligned}
$$

In terms of $\mathrm{CM}$ and relative wave functions, we have

$$
\begin{aligned}
& \frac{e^{i Q(\mathbf{A}(\mathbf{R})-\mathbf{B} \times \mathbf{R}) \cdot \frac{\mathbf{n}_{1 B}+\mathbf{n}_{2 B}}{2} L} \Phi_{E-\varepsilon}\left(\mathbf{R}+\frac{\mathbf{n}_{1 B}+\mathbf{n}_{2 B}}{2} L\right)}{\Phi_{E-\varepsilon}(\mathbf{R})} \\
& =\frac{\psi_{\varepsilon}(\mathbf{r})}{e^{i q(\mathbf{A}(\mathbf{r})-\mathbf{B} \times \mathbf{r}) \cdot\left(\mathbf{n}_{1 B}-\mathbf{n}_{2 B}\right) L} \psi_{\varepsilon}\left(\mathbf{r}+\left(\mathbf{n}_{1 B}-\mathbf{n}_{2 B}\right) L\right)}
\end{aligned}
$$

The separable form of the CM motion and relative motion in Eq. (A25) suggests that both sides must be equal to a phase factor that is independent of both CM and relative coordinates. It allows us to introduce an arbitrary parameter $\mathbf{P}_{B}$ that is associated with a pure translation operator; the phase factor may be chosen having the form of

$$
e^{i \mathbf{P}_{B} \cdot \frac{\mathbf{n}_{1 B}+\mathbf{n}_{2 B}}{2} L} .
$$

Hence the CM wave function satisfies Bloch type magnetic periodic boundary condition,

$\Phi_{E-\varepsilon}\left(\mathbf{R}+\frac{\mathbf{n}_{B} L}{2}\right)=e^{-i\left(\mathbf{P}_{B}+Q B R_{y} \mathbf{e}_{x}\right) \cdot \frac{\mathbf{n}_{B} L}{2}} \Phi_{E-\varepsilon}(\mathbf{R})$.

The boundary condition for a relative wave function is given by

$$
\psi_{\varepsilon}\left(\mathbf{r}+\mathbf{n}_{B} L\right)=e^{i \frac{\mathbf{P}_{B}}{2} \cdot \mathbf{n}_{B} L} e^{-i q B r_{y} \mathbf{e}_{x} \cdot \mathbf{n}_{B} L} \psi_{\varepsilon}(\mathbf{r}),
$$

where we have also assumed

$$
e^{i \mathbf{P}_{B} \cdot \mathbf{n}_{B} L}=1
$$

thus

$$
\mathbf{P}_{B}=2 \pi\left(\frac{n_{x}}{n_{q} L} \mathbf{e}_{x}+\frac{n_{y}}{L} \mathbf{e}_{y}+\frac{n_{z}}{L} \mathbf{e}_{z}\right), \quad n_{x, y, z} \in \mathbb{Z}
$$

Although $\mathbf{P}_{B}$ resembles the total momentum of a system in absence of a magnetic field, $\mathbf{P}_{B}$ is not a conserved quantity in the magnetic field. In fact, the conserved quantity can be identified as a pseudomomentum, see e.g., Ref. [77],

$$
\mathbf{K}_{\mathbf{R}}=\mathbf{P}_{B}+Q(\mathbf{A}(\mathbf{R})-\mathbf{B} \times \mathbf{R})
$$

which is associated with the generator of a magnetic translation operator for CM motion,

$$
\hat{T}_{\mathbf{R}}\left(\frac{\mathbf{n}_{B} L}{2}\right)=e^{i \hat{\mathbf{K}}_{\mathbf{R}} \cdot \frac{\mathbf{n}_{B} L}{2}}, \quad\left[\hat{\mathbf{K}}_{\mathbf{R}}, \hat{H}\right]=0 .
$$

\section{CM motion solutions}

The CM motion of two charged bosons in a uniform magnetic field is described by 


$$
\hat{H}_{\mathbf{R}} \Phi_{E_{R}}(\mathbf{R})=E_{R} \Phi_{E_{R}}(\mathbf{R}),
$$

where

$$
\hat{H}_{\mathbf{R}}=-\frac{1}{2 M}\left[\partial_{R_{x}}^{2}+\left(\partial_{R_{y}}+i Q B R_{x}\right)^{2}+\partial_{R_{z}}^{2}\right]
$$

and $\Phi_{E_{R}}$ must satisfy the boundary condition

$$
\Phi_{E_{R}}\left(\mathbf{R}+\frac{\mathbf{n}_{B} L}{2}\right)=e^{-i\left(\mathbf{P}_{B}+Q B R_{y} \mathbf{e}_{x}\right) \cdot \frac{\mathbf{n}_{B} L}{2}} \Phi_{E_{R}}(\mathbf{R}) .
$$

The solution that satisfies the magnetic periodic boundary condition can be found in [75],

$$
\Phi_{E_{R, n}}(\mathbf{R})=\frac{2}{L} \sum_{k_{y}=\frac{4 \pi n_{y} n_{p}}{L}-P_{B y}}^{n_{y} \in \mathbb{Z}} \phi_{n}\left(R_{x}+\frac{k_{y}}{Q B}\right) e^{i k_{y}\left(R_{y}+\frac{P_{B x}}{Q B}\right)} e^{-i P_{B z} R_{z}},
$$

where $\phi_{n}$ is the eigen-solution of a 1D harmonic oscillator potential,

$$
-\frac{1}{2 M}\left[\partial_{R_{x}}^{2}-(Q B)^{2} R_{x}^{2}\right] \phi_{n}\left(R_{x}\right)=\frac{Q B}{M}\left(n+\frac{1}{2}\right) \phi_{n}\left(R_{x}\right) .
$$

The eigen-energy of CM motion is given by

$$
E_{R, n}=\frac{Q B}{M}\left(n+\frac{1}{2}\right)+\frac{P_{B z}^{2}}{2 M}, \quad n=0,1,2, \ldots,
$$

and the analytic expression of $\phi_{n}$ is

$$
\phi_{n}\left(R_{x}\right)=\frac{1}{\sqrt{2^{n} n !}}\left(\frac{Q B}{\pi}\right)^{\frac{1}{4}} e^{-\frac{Q B}{2} R_{x}^{2}} H_{n}\left(\sqrt{Q B} R_{x}\right) .
$$

\section{Relative motion and finite volume Lippmann-Schwinger equation}

The relative motion of two charged particles in a uniform magnetic field is described by

$$
\left(\hat{H}_{\mathbf{r}}+V(\mathbf{r})\right) \psi_{\varepsilon}(\mathbf{r})=\varepsilon \psi_{\varepsilon}(\mathbf{r}),
$$

where

$$
\hat{H}_{\mathbf{r}}=-\frac{1}{2 \mu}\left[\partial_{r_{x}}^{2}+\left(\partial_{r_{y}}+i q B r_{x}\right)^{2}+\partial_{r_{z}}^{2}\right]
$$

and $\psi_{\varepsilon}$ must satisfy the magnetic periodic boundary condition

$$
\psi_{\varepsilon}\left(\mathbf{r}+\mathbf{n}_{B} L\right)=e^{i \frac{\mathbf{p}_{B}}{2} \cdot \mathbf{n}_{B} L} e^{-i q B r_{y} \mathbf{e}_{x} \cdot \mathbf{n}_{B} L} \psi_{\varepsilon}(\mathbf{r}) .
$$

The integral representation of Schrödinger Eq. (A38) and magnetic periodic boundary condition in Eq. (A40) together is given by the finite volume Lippmann-Schwinger equation,

$$
\psi_{\varepsilon}(\mathbf{r})=\int_{L_{B}^{3}} d \mathbf{r}^{\prime} G_{B}^{(L)}\left(\mathbf{r}, \mathbf{r}^{\prime} ; \varepsilon\right) V\left(\mathbf{r}^{\prime}\right) \psi_{\varepsilon}\left(\mathbf{r}^{\prime}\right),
$$

where $L_{B}^{3}$ stands for the volume of the magnetic unit box defined by unit vectors

$$
n_{q} L \mathbf{e}_{x} \times L \mathbf{e}_{y} \times L \mathbf{e}_{z},
$$

and

$$
\int_{L_{B}^{3}} d \mathbf{r}^{\prime}=\int_{-\frac{n_{q} L}{2}}^{\frac{n_{q} L}{2}} d r_{x}^{\prime} \int_{-\frac{L}{2}}^{\frac{L}{2}} d r_{y}^{\prime} \int_{-\frac{L}{2}}^{\frac{L}{2}} d r_{z}^{\prime}
$$

The finite volume magnetic Green's function $G_{B}^{(L)}$ also must satisfy the magnetic periodic boundary condition,

$$
\begin{aligned}
& G_{B}^{(L)}\left(\mathbf{r}, \mathbf{r}^{\prime} ; \varepsilon\right) \\
& \quad=e^{-i \frac{\mathbf{P}_{B}}{2} \cdot \mathbf{n}_{B} L} e^{i q B r_{y} \mathbf{e}_{x} \cdot \mathbf{n}_{B} L} G_{B}^{(L)}\left(\mathbf{r}+\mathbf{n}_{B} L, \mathbf{r}^{\prime} ; \varepsilon\right) \\
& \quad=e^{\mathbf{P}_{B} \frac{\mathbf{P}_{B}}{2} \mathbf{n}_{B} L} e^{-i q B r_{y}^{\prime} \mathbf{e}_{x} \cdot \mathbf{n}_{B} L} G_{B}^{(L)}\left(\mathbf{r}, \mathbf{r}^{\prime}+\mathbf{n}_{B} L ; \varepsilon\right) .
\end{aligned}
$$

The magnetic periodic boundary conditions and Eq. (A41) suggest that $G_{B}^{(L)}$ is the solution of the differential equation,

$$
\begin{aligned}
& \left(\varepsilon-\hat{H}_{\mathbf{r}}\right) G_{B}^{(L)}\left(\mathbf{r}, \mathbf{r}^{\prime} ; \varepsilon\right) \\
& \quad=\sum_{\mathbf{n}_{B}} e^{-i \frac{\mathbf{P}_{B}}{2} \cdot \mathbf{n}_{B} L} e^{i q B r_{y} \mathbf{e}_{x} \cdot \mathbf{n}_{B} L} \delta\left(\mathbf{r}-\mathbf{r}^{\prime}+\mathbf{n}_{B} L\right) .
\end{aligned}
$$

Now, one of the key steps therefore is to find an analytic solution of finite volume magnetic Green's function $G_{B}^{(L)}$. The $G_{B}^{(L)}$ can be constructed from an infinite volume magnetic Green's function $G_{B}^{(\infty)}$, where

$$
\begin{aligned}
G_{B}^{(\infty)}\left(\mathbf{r}, \mathbf{r}^{\prime} ; \varepsilon\right)= & \sum_{n=0}^{\infty} \int_{-\infty}^{\infty} \frac{d k_{y}}{2 \pi} \frac{d k_{z}}{2 \pi} \\
& \times \frac{\phi_{n}\left(r_{x}+\frac{k_{y}}{q B}\right) \phi_{n}^{*}\left(r_{x}^{\prime}+\frac{k_{y}}{q B}\right) e^{i k_{y}\left(r_{y}-r_{y}^{\prime}\right)} e^{i k_{z}\left(r_{z}-r_{z}^{\prime}\right)}}{\varepsilon-\frac{q B}{\mu}\left(n+\frac{1}{2}\right)-\frac{k_{z}^{2}}{2 \mu}},
\end{aligned}
$$

and $G_{B}^{(\infty)}$ satisfies the equation,

$$
\left(\varepsilon-\hat{H}_{\mathbf{r}}\right) G_{B}^{(\infty)}\left(\mathbf{r}, \mathbf{r}^{\prime} ; \varepsilon\right)=\delta\left(\mathbf{r}-\mathbf{r}^{\prime}\right)
$$


The LS equation (A41) is equivalently given in terms of $G_{B}^{(\infty)}$ by

$$
\psi_{\varepsilon}(\mathbf{r})=\int_{-\infty}^{\infty} d \mathbf{r}^{\prime} G_{B}^{(\infty)}\left(\mathbf{r}, \mathbf{r}^{\prime} ; \varepsilon\right) V\left(\mathbf{r}^{\prime}\right) \psi_{\varepsilon}\left(\mathbf{r}^{\prime}\right)
$$

The integration over infinite volume in Eq. (A47) can be folded up to an infinite sum of integration in a magnetic cell,

$$
\begin{aligned}
\psi_{\varepsilon}(\mathbf{r})= & \sum_{\mathbf{n}_{B}} \int_{L_{B}^{3}} d \mathbf{r}^{\prime} G_{B}^{(\infty)}\left(\mathbf{r}, \mathbf{r}^{\prime}+\mathbf{n}_{B} L ; \varepsilon\right) \\
& \times V\left(\mathbf{r}^{\prime}+\mathbf{n}_{B} L\right) \psi_{\varepsilon}\left(\mathbf{r}^{\prime}+\mathbf{n}_{B} L\right)
\end{aligned}
$$

Using the magnetic periodic boundary condition given in Eq. (A40), $G_{B}^{(L)}$ is thus identified as

$$
\begin{aligned}
G_{B}^{(L)}\left(\mathbf{r}, \mathbf{r}^{\prime} ; \varepsilon\right) & =\sum_{\mathbf{n}_{B}} G_{B}^{(\infty)}\left(\mathbf{r}, \mathbf{r}^{\prime}+\mathbf{n}_{B} L ; \varepsilon\right) e^{i \frac{\mathbf{P}_{B}}{2} \cdot \mathbf{n}_{B} L} e^{-i q B r_{y}^{\prime} \mathbf{e}_{x} \cdot \mathbf{n}_{B} L} \\
& =\sum_{\mathbf{n}_{B}} e^{-i \frac{\mathbf{P}_{B}}{2} \cdot \mathbf{n}_{B} L} e^{i q B r_{y} \mathbf{e}_{x} \cdot \mathbf{n}_{B} L} G_{B}^{(\infty)}\left(\mathbf{r}+\mathbf{n}_{B} L, \mathbf{r}^{\prime} ; \varepsilon\right) .
\end{aligned}
$$

Hence, explicitly we find

$$
\begin{aligned}
G_{B}^{(L)}\left(\mathbf{r}, \mathbf{r}^{\prime} ; \varepsilon\right)= & \sum_{n_{x} \in \mathbb{Z}} e^{-i\left(\frac{P_{B x}}{2}-q B r_{y}\right) n_{x} n_{q} L} \\
& \times \frac{1}{L^{2}} \sum_{k_{y, z}=\frac{2 \pi n_{y, z}}{L}+\frac{P_{B y, B z}}{n_{y, z} \in \mathbb{Z}}} e^{i k_{y}\left(r_{y}-r_{y}^{\prime}\right)} e^{i k_{z}\left(r_{z}-r_{z}^{\prime}\right)} \\
& \times \sum_{n=0}^{\infty} \frac{\phi_{n}\left(r_{x}+n_{x} n_{q} L+\frac{k_{y}}{q B}\right) \phi_{n}^{*}\left(r_{x}^{\prime}+\frac{k_{y}}{q B}\right)}{\varepsilon-\frac{q B}{\mu}\left(n+\frac{1}{2}\right)-\frac{k_{z}^{2}}{2 \mu}} .
\end{aligned}
$$

The other representation of $G_{B}^{(\infty)}$ is given in Refs. [78,79] by

$$
\begin{aligned}
G_{B}^{(\infty)}\left(\mathbf{r}, \mathbf{r}^{\prime} ; \varepsilon\right)= & e^{-\frac{i q B}{2}\left(r_{x}+r_{x}^{\prime}\right)\left(r_{y}-r_{y}^{\prime}\right)} e^{-\frac{q B}{4}\left|\boldsymbol{\rho}-\boldsymbol{\rho}^{\prime}\right|^{2}} \\
& \times \frac{q B}{2 \pi} \int_{-\infty}^{\infty} \frac{d k_{z}}{2 \pi} \sum_{n=0}^{\infty} \frac{L_{n}\left(\frac{q B}{2}\left|\boldsymbol{\rho}-\boldsymbol{\rho}^{\prime}\right|^{2}\right) e^{i k_{z}\left(r_{z}-r_{z}^{\prime}\right)}}{\varepsilon-\frac{q B}{\mu}\left(n+\frac{1}{2}\right)-\frac{k_{z}^{2}}{2 \mu}},
\end{aligned}
$$

where
Therefore, $G_{B}^{(L)}$ is also given by

$$
\begin{aligned}
& G_{B}^{(L)}\left(\mathbf{r}, \mathbf{r}^{\prime} ; \varepsilon\right) \\
& =\sum_{n_{x}, n_{y} \in \mathbb{Z}} e^{-i\left(\frac{P_{B x}}{2}-q B r_{y}\right) n_{x} n_{q} L} e^{-i \frac{P_{B y}}{2} n_{y} L} e^{-\frac{i q B}{2}\left(r_{x}+r_{x}^{\prime}+n_{x} n_{q} L\right)\left(r_{y}-r_{y}^{\prime}+n_{y} L\right)} \\
& \times e^{-\frac{q B}{4}\left|\boldsymbol{\rho}-\boldsymbol{\rho}^{\prime}+n_{x} n_{q} L \mathbf{e}_{x}+n_{y} L \mathbf{e}_{y}\right|^{2}} \frac{q B}{2 \pi} \frac{1}{L} \sum_{k_{z}=\frac{2 \pi n_{z}}{L}+\frac{P_{B z}}{2}}^{n_{z} \in \mathbb{Z}} \\
& \quad \times \sum_{n=0}^{\infty} \frac{L_{n}\left(\frac{q B}{2}\left|\boldsymbol{\rho}-\boldsymbol{\rho}^{\prime}+n_{x} n_{q} L \mathbf{e}_{x}+n_{y} L \mathbf{e}_{y}\right|^{2}\right) e^{i k_{z}\left(r_{z}-r_{z}^{\prime}\right)}}{\varepsilon-\frac{q B}{\mu}\left(n+\frac{1}{2}\right)-\frac{k_{z}^{2}}{2 \mu}}
\end{aligned}
$$

\section{APPENDIX B: CONNECTING BOUND STATES IN A TRAP TO INFINITE VOLUME SCATTERING STATE}

In this Appendix, we present a general formalism and discussion on the topic of building connections between a discrete energy spectrum of bound state in a trap and infinite volume scattering dynamics. The type of trap is not specified in what follows; the typical and commonly used traps are periodic finite box in LQCD, harmonic potential in nuclear physics, etc.

The relative motion of two interacting particles in a trap is described by the Schrödinger equation

$$
\hat{H}_{\text {trap }} \psi_{\varepsilon}^{(\text {trap })}(\mathbf{r})+\int_{\text {trap }} d \mathbf{r}^{\prime} V\left(\mathbf{r}, \mathbf{r}^{\prime}\right) \psi_{\varepsilon}^{(\text {trap })}\left(\mathbf{r}^{\prime}\right)=\varepsilon \psi_{\varepsilon}^{(\text {trap })}(\mathbf{r}),
$$

where $\hat{H}_{\text {trap }}$ stands for the trap Hamiltonian operator, and the interaction between particles is described by a nonlocal short-range interaction $V\left(\mathbf{r}, \mathbf{r}^{\prime}\right)$ in general. The effect of a trap is usually reflected by both the trap Hamiltonian and boundary condition of the wave function in a trap. In the case of charged particles trapped in both a periodic box and a uniform magnetic field, $\hat{H}_{\text {trap }}$ and the boundary condition are thus given by $\hat{H}_{\mathbf{r}}$ in Eq. (A39) and magnetic periodic boundary condition in Eq. (A40) respectively. The energy spectrum hence becomes discrete.

In infinite volume, the dynamics of two interacting particles through the same short-range interaction $V\left(\mathbf{r}, \mathbf{r}^{\prime}\right)$ is given by

$$
\hat{H}_{0} \psi_{\varepsilon_{\infty}}^{(\infty)}(\mathbf{r})+\int_{-\infty}^{\infty} d \mathbf{r}^{\prime} V\left(\mathbf{r}, \mathbf{r}^{\prime}\right) \psi_{\varepsilon_{\infty}}^{(\infty)}\left(\mathbf{r}^{\prime}\right)=\varepsilon_{\infty} \psi_{\varepsilon_{\infty}}^{(\infty)}(\mathbf{r}),
$$

where

$$
\boldsymbol{\rho}=r_{x} \mathbf{e}_{x}+r_{y} \mathbf{e}_{y}, \quad \boldsymbol{\rho}^{\prime}=r_{x}^{\prime} \mathbf{e}_{x}+r_{y}^{\prime} \mathbf{e}_{y} .
$$




$$
\hat{H}_{0}=-\frac{\nabla_{\mathbf{r}}^{2}}{2 \mu}
$$

The energy spectrum of the scattering solution in infinite volume is continuous. With an incoming plane wave,

$$
e^{i \mathbf{q} \cdot \mathbf{r}} \quad \text { where } q=\sqrt{2 \mu \varepsilon_{\infty}},
$$

the asymptotic wave function of scattering states is thus described by on-shell scattering amplitudes,

$\psi_{\varepsilon_{\infty}}^{(\infty)}(\mathbf{r}) \stackrel{r \rightarrow \infty}{\rightarrow} \sum_{l}(2 l+1) P_{l}(\hat{\mathbf{q}} \cdot \hat{\mathbf{r}}) i^{l}\left[j_{l}(q r)+i t_{l}(q) h_{l}^{(+)}(q r)\right]$,

where $t_{l}(q)$ denotes the elastic on-shell partial wave scattering amplitude and can be parametrized by a phase shift function $\delta_{l}(q)$,

$$
t_{l}(q)=\frac{1}{\cot \delta_{l}(q)-i} .
$$

We also remark that in a general case, depending on the trap, the infinite volume relative energy $\varepsilon_{\infty}$ is related to finite volume relative energy $\varepsilon$ by the shared total energy. For instance, in the case of charged particles trapped in both a periodic box and a uniform magnetic field,

$$
\varepsilon_{\infty}+\frac{\mathbf{P}^{2}}{2 M}=\varepsilon+E_{R, n}=E,
$$

where CM energy $E_{R, n}$ is given by Eq. (A36).

The dynamics of particles in a trap and in infinite volume are associated by the short-range interaction potential between two particles. As far as the range of potential is far smaller than the size of the trap, a compact expression between phase shift of scattering states and a function, $\mathcal{M}_{l m, l^{\prime} m^{\prime}}(\varepsilon)$, that reflect geometric and dynamical properties of the trap can be found,

$$
\operatorname{det}\left[\delta_{l m, l^{\prime} m^{\prime}} \cot \delta_{l}(q)-\mathcal{M}_{l m, l^{\prime} m^{\prime}}(\varepsilon)\right]=0 .
$$

In the case of finite volume in LQCD, this relation is a wellknown Lüscher formula [1], the matrix function $\mathcal{M}_{l m, l^{\prime} m^{\prime}}(\varepsilon)$ is thus a zeta function. In finite volume, the angular momentum is no longer a good quantum number due to the breaking rotation symmetry in finite volume. In the case of harmonic trap in nuclear physics, the relation is known as the BERW formula [42-51], where the function $\mathcal{M}_{l m, l^{\prime} m^{\prime}}$ becomes diagonal in angular momentum basis. The simple form of the quantization condition in Eq. (B6) is the result of the presence of two distinguishable scales: (1) short-range interaction between two particles and (2) size of trap. Hence the short-range dynamics that is described by phase shift or scattering amplitude and long- range physics due to the presence of a trap can be factorized.

The derivation of tje Lüscher formula or BERW formula can be illustrated by considering momentum space Lippmann-Schwinger equation under the assumption of separable potential, see e.g., [28-30], and an example of derivation of BERW formula in momentum space is given in Appendix C. Here the result is only summarized briefly symbolically; the reaction amplitudes in both trap and infinite volume may be introduced respectively by

$$
\hat{t}_{\text {trap }}=-\hat{V} \hat{\psi} \quad \text { and } \quad \hat{t}_{\infty}=-\hat{V} \hat{\psi}_{\infty},
$$

They satisfy integral LS equations,

$$
\hat{t}_{\text {trap }}(\varepsilon)=\hat{V} \hat{G}_{\text {trap }}(\varepsilon) \hat{t}_{\text {trap }}(\varepsilon),
$$

and

$$
\hat{t}_{\infty}(q)=-\hat{V}+\hat{V} \hat{G}_{\infty}(q) \hat{t}_{\infty}(q)
$$

where

$$
\hat{G}_{\text {trap }}(\varepsilon)=\frac{1}{\varepsilon-\hat{H}_{\text {trap }}}
$$

and

$$
\hat{G}_{\infty}(q)=\frac{1}{\frac{q^{2}}{2 \mu}-\hat{H}_{0}}
$$

are Green's function in a trap and in infinite volume respectively. Under the assumption of a separable potential that is equivalent to the zero-range interaction,

$$
\tilde{V}\left(\mathbf{k}, \mathbf{k}^{\prime}\right)=\sum_{l m}\left(k k^{\prime}\right)^{l} V_{l} Y_{l m}(\hat{\mathbf{k}}) Y_{l m}^{*}\left(\hat{\mathbf{k}}^{\prime}\right),
$$

Eq. (B7) and Eq. (B8) are turned into algebraic equations, and can be solved analytically [30]. Eliminating $\hat{V}$ from two equations, the quantization condition is thus obtained

$$
\operatorname{det}\left[\frac{1}{\hat{t}_{\infty}(q)}-\hat{G}_{\infty}(q)+\hat{G}_{\text {trap }}(\varepsilon)\right]=0,
$$

which is equivalent to Eq. (B6).

Though the plane wave basis in momentum space may be a very convenient basis in finite volume and other types of traps, for the charged particles in a uniform magnetic field, the momentum is no longer the conserved quantity due to the breaking translation symmetry by magnetic field. Introducing a reaction amplitude in momentum space becomes a tricky business. Therefore, in what follows, instead of working in momentum space, we will present the general discussion of derivation of a quantization condition 
in coordinate space under the assumption of a separable short-range potential again. The Fourier transform of a separable potential given in Eq. (B11) is

$$
\begin{aligned}
V\left(\mathbf{r}, \mathbf{r}^{\prime}\right) & =\int \frac{d \mathbf{k}}{(2 \pi)^{3}} \frac{d \mathbf{k}^{\prime}}{(2 \pi)^{3}} e^{-i \mathbf{k} \cdot \mathbf{r}} \tilde{V}\left(\mathbf{k}, \mathbf{k}^{\prime}\right) e^{i \mathbf{k}^{\prime} \cdot \mathbf{r}^{\prime}} \\
& =\frac{\delta(r) \delta\left(r^{\prime}\right)}{\left(r r^{\prime}\right)^{2}} \sum_{l m} V_{l} \frac{2^{2 l+1} \Gamma^{2}\left(l+\frac{3}{2}\right)}{(2 \pi)^{3}\left(r r^{\prime}\right)^{l}} Y_{l m}(\hat{\mathbf{r}}) Y_{l m}^{*}\left(\hat{\mathbf{r}}^{\prime}\right) .
\end{aligned}
$$

\section{Dynamical equation in a trap}

In the trap, the integral representation of Eq. (B1) is given by the Lippmann-Schwinger equation

$$
\begin{aligned}
\psi_{\varepsilon}^{(\text {trap })}(\mathbf{r})= & \int_{\text {trap }} d \mathbf{r}^{\prime \prime} G^{(\text {trap })}\left(\mathbf{r}, \mathbf{r}^{\prime \prime} ; \varepsilon\right) \\
& \times \int_{\text {trap }} d \mathbf{r}^{\prime} V\left(\mathbf{r}^{\prime \prime}, \mathbf{r}^{\prime}\right) \psi_{\varepsilon}^{(\text {trap })}\left(\mathbf{r}^{\prime}\right),
\end{aligned}
$$

where

$$
G^{(\text {trap })}\left(\mathbf{r}, \mathbf{r}^{\prime \prime} ; \varepsilon\right)=\left\langle\mathbf{r}\left|\frac{1}{\varepsilon-\hat{H}_{\text {trap }}}\right| \mathbf{r}^{\prime \prime}\right\rangle
$$

stands for the Green's function in a trap. The partial wave expansions

$$
\psi_{\varepsilon}^{(\text {trap })}(\mathbf{r})=\sum_{l m} \psi_{l m}^{(\text {trap })}(r) Y_{l m}(\hat{\mathbf{r}})
$$

and

$$
G^{(t r a p)}\left(\mathbf{r}, \mathbf{r}^{\prime \prime} ; \varepsilon\right)=\sum_{l m, l^{\prime \prime} m^{\prime \prime}} Y_{l m}(\hat{\mathbf{r}}) G_{l m, l^{\prime \prime} m^{\prime \prime}}^{(t r a p)}\left(r, r^{\prime \prime} ; \varepsilon\right) Y_{l^{\prime \prime} m^{\prime \prime}}^{*}\left(\hat{\mathbf{r}}^{\prime \prime}\right)
$$

yield

$$
\begin{aligned}
\psi_{l m}^{(\text {trap })}(r)= & \sum_{l^{\prime} m^{\prime}} \int_{\text {trap }} r^{\prime \prime 2} d r^{\prime \prime} G_{l m, l^{\prime} m^{\prime}}^{(t r a p)}\left(r, r^{\prime \prime} ; \varepsilon\right) \\
& \times \int_{\text {trap }} r^{\prime 2} d r^{\prime} V_{l^{\prime}}\left(r^{\prime \prime}, r^{\prime}\right) \psi_{l^{\prime} m^{\prime}}^{(t r a p)}\left(r^{\prime}\right) .
\end{aligned}
$$

Under the assumption of a separable potential with the form of Eq. (B13), Eq. (B18) is turned into an algebraic equation,

$$
\begin{aligned}
\frac{\psi_{l m}^{(\text {trap })}(r)}{r^{l}}= & \sum_{l^{\prime} m^{\prime}} V_{l^{\prime}} \frac{2^{2 l^{\prime}+1} \Gamma^{2}\left(l^{\prime}+\frac{3}{2}\right)}{(2 \pi)^{3}} \\
& \times\left.\frac{G_{l m, l^{\prime} m^{\prime}}^{(t r a p)}\left(r, r^{\prime \prime} ; \varepsilon\right)}{r^{l} r^{\prime \prime l^{\prime}}} \frac{\psi_{l^{\prime} m^{\prime}}^{(t r a p)}\left(r^{\prime}\right)}{r^{\prime l^{\prime}}}\right|_{r^{\prime}, r^{\prime \prime} \rightarrow 0} .
\end{aligned}
$$

hence the discrete energy spectrum is determined by

$\operatorname{det}\left[\frac{\delta_{l m, l^{\prime} m^{\prime}}}{2^{2 l^{\prime}+1} V_{l}}-\left.\frac{\Gamma^{2}\left(l^{\prime}+\frac{3}{2}\right)}{(2 \pi)^{3}} \frac{G_{l m, l^{\prime} m^{\prime}}^{(t r a p)}\left(r, r^{\prime} ; \varepsilon\right)}{r^{l} r^{\prime l^{\prime}}}\right|_{r, r^{\prime} \rightarrow 0}\right]=0$.

\section{Infinite volume dynamical equation}

In infinite volume, with an incoming plane wave of $e^{i \mathbf{q} \cdot \mathbf{r}}$, the scattering solution of two particles interaction is described by inhomogeneous integral LippmannSchwinger equation,

$\psi_{\varepsilon_{\infty}}^{(\infty)}(\mathbf{r}, \mathbf{q})$

$=e^{i \mathbf{q} \cdot \mathbf{r}}+\int_{-\infty}^{\infty} d \mathbf{r}^{\prime \prime} G^{(\infty)}\left(\mathbf{r}-\mathbf{r}^{\prime \prime} ; q\right) \int_{-\infty}^{\infty} d \mathbf{r}^{\prime} V\left(\mathbf{r}^{\prime \prime}, \mathbf{r}^{\prime}\right) \psi_{\varepsilon_{\infty}}^{(\infty)}\left(\mathbf{r}^{\prime}, \mathbf{q}\right)$,

where $q=\sqrt{2 \mu \varepsilon_{\infty}}$, and the Green's function is given by

$$
\begin{aligned}
G^{(\infty)} & \left(\mathbf{r}-\mathbf{r}^{\prime \prime} ; q\right) \\
& =\int \frac{d \mathbf{p}}{(2 \pi)^{3}} \frac{e^{i \mathbf{p} \cdot\left(\mathbf{r}-\mathbf{r}^{\prime \prime}\right)}}{\frac{q^{2}}{2 \mu}-\frac{\mathbf{p}^{2}}{2 \mu}}=-\frac{2 \mu}{4 \pi} i q h_{0}^{(+)}\left(q\left|\mathbf{r}-\mathbf{r}^{\prime \prime}\right|\right)
\end{aligned}
$$

Considering partial wave expansion,

$$
\psi_{\varepsilon_{\infty}}^{(\infty)}(\mathbf{r}, \mathbf{q})=\sum_{l m} Y_{l m}^{*}(\hat{\mathbf{q}}) \psi_{l}^{(\infty)}(r, q) Y_{l m}(\hat{\mathbf{r}}),
$$

and

$$
\begin{aligned}
G^{(\infty)}\left(\mathbf{r}-\mathbf{r}^{\prime \prime} ; q\right) & =\sum_{l m} Y_{l m}(\hat{\mathbf{r}}) G_{l}^{(\infty)}\left(r, r^{\prime \prime} ; q\right) Y_{l m}^{*}\left(\hat{\mathbf{r}}^{\prime \prime}\right) \\
G_{l}^{(\infty)}\left(r, r^{\prime \prime} ; q\right) & =-2 \mu i q j_{l}\left(q r_{<}\right) h_{l}^{(+)}\left(q r_{>}\right)
\end{aligned}
$$

we thus obtain

$$
\begin{aligned}
\psi_{l}^{(\infty)}(r, q)= & 4 \pi i^{l} j_{l}(q r)+\int_{0}^{\infty} r^{\prime \prime 2} d r^{\prime \prime} G_{l}^{(\infty)}\left(r, r^{\prime \prime} ; q\right) \\
& \times \int_{0}^{\infty} r^{\prime 2} d r^{\prime} V_{l}\left(r^{\prime \prime}, r^{\prime}\right) \psi_{l}^{(\infty)}\left(r^{\prime}, q\right) .
\end{aligned}
$$


The separable potential given in Eq. (B13) yields an algebraic equation

$$
\begin{aligned}
& \frac{\psi_{l}^{(\infty)}(r, q)}{r^{l}}=4 \pi i^{l} \frac{j_{l}(q r)}{r^{l}} \\
& \quad+\left.V_{l} \frac{2^{2 l+1} \Gamma^{2}\left(l+\frac{3}{2}\right)}{(2 \pi)^{3}} \frac{G_{l}^{(\infty)}\left(r, r^{\prime \prime} ; q\right)}{\left(r r^{\prime \prime}\right)^{l}} \frac{\psi_{l}^{(\infty)}\left(r^{\prime}, q\right)}{r^{\prime l}}\right|_{r^{\prime}, r^{\prime \prime} \rightarrow 0} .
\end{aligned}
$$

The wave function solution is thus given by

$$
\frac{\psi_{l}^{(\infty)}(r, q)}{r^{l}}=4 \pi i^{l}\left[\frac{j_{l}(q r)}{r^{l}}+i t_{l}(q) \frac{h_{l}^{(+)}(q r)}{r^{l}}\right],
$$

where the partial wave two-body scattering amplitude $t_{l}(q)$ is given by

$$
t_{l}(q)=-\frac{\frac{2 \mu q^{2 l+1}}{(4 \pi)^{2}}}{\frac{1}{V_{l}}-\left.\frac{2^{2 l+1} \Gamma^{2}\left(l+\frac{3}{2}\right)}{(2 \pi)^{3}} \frac{G_{l}^{(\infty)}\left(r^{\prime}, r^{\prime \prime} ; q\right)}{\left(r^{\prime} r^{\prime \prime}\right)^{l}}\right|_{r^{\prime}, r^{\prime \prime} \rightarrow 0}} .
$$

\section{Quantization condition in a trap}

Combining Eqs. (B20) and (B28), and eliminating $V_{l}$, one thus find

$$
\begin{aligned}
\operatorname{det} & {\left[\delta_{l m, l^{\prime} m^{\prime}} \frac{2 \mu q^{2 l+1}}{t_{l}(q)}\right.} \\
& -\left.\delta_{l m, l^{\prime} m^{\prime}} \frac{2^{2 l+3} \Gamma^{2}\left(l+\frac{3}{2}\right)}{(2 \pi)} \frac{G_{l}^{(\infty)}\left(r, r^{\prime} ; q\right)}{\left(r r^{\prime}\right)^{l}}\right|_{r, r^{\prime} \rightarrow 0} \\
& \left.+\left.\frac{2^{2 l^{\prime}+3} \Gamma^{2}\left(l^{\prime}+\frac{3}{2}\right)}{(2 \pi)} \frac{G_{l m, l^{\prime} m^{\prime}}^{(t r a p)}\left(r, r^{\prime} ; \varepsilon\right)}{r^{l} r^{\prime l^{\prime}}}\right|_{r, r^{\prime} \rightarrow 0}\right]=0 .
\end{aligned}
$$

Using the asymptotic form of

$$
\begin{aligned}
& \left.\frac{2^{2 l+3} \Gamma^{2}\left(l+\frac{3}{2}\right)}{2 \mu(2 \pi)} \frac{G_{l}^{(\infty)}\left(r, r^{\prime} ; q\right)}{\left(r r^{\prime}\right)^{l}}\right|_{r, r^{\prime} \rightarrow 0} \\
& =-i q^{2 l+1}-\left.\frac{2^{2 l+1} \Gamma\left(l+\frac{1}{2}\right) \Gamma\left(l+\frac{3}{2}\right)}{\pi} \frac{1}{r^{2 l+1}}\right|_{r \rightarrow 0},
\end{aligned}
$$

and also the parameterization of

$$
t_{l}^{-1}(q)=\cot \delta_{l}(q)-i
$$

thus the quantization condition in a trap is indeed given by a Lüscher formulalike relation,

$$
\operatorname{det}\left[\delta_{l m, l^{\prime} m^{\prime}} \cot \delta_{l}(q)-\mathcal{M}_{l m, l^{\prime} m^{\prime}}(\varepsilon)\right]=0
$$

where

$$
\begin{aligned}
\mathcal{M}_{l m, l^{\prime} m^{\prime}}(\varepsilon)= & -\left.\frac{2^{2 l^{\prime}+3} \Gamma^{2}\left(l^{\prime}+\frac{3}{2}\right)}{2 \mu q^{2 l+1}(2 \pi)} \frac{G_{l m, l^{\prime} m^{\prime}}^{(t r a p)}\left(r, r^{\prime} ; \varepsilon\right)}{r^{l} r^{\prime l^{\prime}}}\right|_{r, r^{\prime} \rightarrow 0} \\
& -\left.\delta_{l m, l^{\prime} m^{\prime}} \frac{2^{2 l+1} \Gamma\left(l+\frac{1}{2}\right) \Gamma\left(l+\frac{3}{2}\right)}{\pi} \frac{1}{(q r)^{2 l+1}}\right|_{r \rightarrow 0} .
\end{aligned}
$$

The second term in Eq. (B32) is an ultraviolet counterterm that would cancel out the ultraviolet divergent term in $G_{l m, l^{\prime} m^{\prime}}^{(t r a p)}$, and ultimate result is finite and well defined.

\section{APPENDIX C: MOMENTUM SPACE LS EQUATION AND PARTICLES INTERACTION IN A HARMONIC TRAP}

In this section, we present some technical details of nonrelativistic spinless particles interaction in a harmonic trap. The dynamics of nonrelativistic bosonic particles interaction in a harmonic trap is described by

$$
\left(\hat{H}^{(h o)}+\hat{V}\right) \Psi_{E}^{(h o)}\left(\mathbf{x}_{1}, \mathbf{x}_{2}\right)=E \Psi_{E}^{(h o)}\left(\mathbf{x}_{1}, \mathbf{x}_{2}\right),
$$

where

$$
\hat{H}^{(h o)}=\sum_{i=1}^{2}\left(-\frac{\nabla_{i}^{2}}{2 m}+\frac{1}{2} m \omega^{2} \mathbf{x}_{i}^{2}\right),
$$

and $\mathbf{x}_{i}$ again stands for the $i$ th particle's position, the $\hat{V}$ represents the interaction between two particles. $\omega$ is the angular frequency of the oscillator. The separation of CM and relative motions

$$
\hat{H}^{(h o)}=\hat{H}_{\mathbf{R}}^{(h o)}+\hat{H}_{\mathbf{r}}^{(h o)},
$$

where

$$
\hat{H}_{\mathbf{R}}^{(h o)}=-\frac{\nabla_{\mathbf{R}}^{2}}{2 M}+\frac{1}{2} M \omega^{2} \mathbf{R}^{2}
$$

and

$$
\hat{H}_{\mathbf{r}}^{(h o)}=-\frac{\nabla_{\mathbf{r}}^{2}}{2 \mu}+\frac{1}{2} \mu \omega^{2} \mathbf{r}^{2}
$$

yield again

$$
\Psi_{E}^{(h o)}\left(\mathbf{x}_{1}, \mathbf{x}_{2}\right)=\Phi_{E_{R, n}}^{(h o)}(\mathbf{R}) \psi_{\varepsilon}^{(h o)}(\mathbf{r}) .
$$

The CM wave function $\Phi_{E_{R, n}}^{(h o)}(\mathbf{R})$ is the solution of the 3D harmonic oscillator potential, 


$$
\hat{H}_{\mathbf{R}}^{(h o)} \Phi_{E_{R, n}}^{(h o)}(\mathbf{R})=E_{R, n} \Phi_{E_{R, n}}^{(h o)}(\mathbf{R}),
$$

where eigen-energy is given by

$$
E_{R, n}=\omega\left(n+\frac{3}{2}\right), \quad n=0,1,2, \ldots
$$

The relative wave function $\psi_{\varepsilon}^{(h o)}(\mathbf{r})$ satisfies the Lippmann-Schwinger equation,

$$
\psi_{\varepsilon}^{(h o)}(\mathbf{r})=\int d \mathbf{r}^{\prime} G^{(h o)}\left(\mathbf{r}, \mathbf{r}^{\prime} ; \varepsilon\right) \int d \mathbf{r}^{\prime \prime} V\left(\mathbf{r}^{\prime}, \mathbf{r}^{\prime \prime}\right) \psi_{\varepsilon}^{(h o)}\left(\mathbf{r}^{\prime \prime}\right),
$$

where Green's function satisfies the equation

$$
\left(\varepsilon-\hat{H}_{\mathbf{r}}^{(h o)}\right) G^{(h o)}\left(\mathbf{r}, \mathbf{r}^{\prime} ; \varepsilon\right)=\delta\left(\mathbf{r}-\mathbf{r}^{\prime}\right) .
$$

The analytic expression of Green's function in a harmonic trap is given by [80]

$$
\begin{aligned}
G^{(h o)}\left(\mathbf{r}, \mathbf{r}^{\prime} ; \varepsilon\right)= & \sum_{l m} Y_{l m}(\hat{\mathbf{r}}) G_{l}^{(h o)}\left(r, r^{\prime} ; \varepsilon\right) Y_{l m}^{*}\left(\hat{\mathbf{r}}^{\prime}\right), \\
G_{l}^{(h o)}\left(r, r^{\prime} ; \varepsilon\right)= & -\frac{1}{\omega\left(r r^{\prime}\right)^{\frac{3}{2}}} \frac{\Gamma\left(\frac{l}{2}+\frac{3}{4}-\frac{\varepsilon}{2 \omega}\right)}{\Gamma\left(l+\frac{3}{2}\right)} \\
& \times \mathcal{M}_{\frac{\varepsilon}{2 \omega}, \frac{l}{2}+\frac{1}{4}}\left(\mu \omega r_{<}^{2}\right) \mathcal{W}_{\frac{\varepsilon}{2 \omega}, \frac{l}{2}+\frac{1}{4}}\left(\mu \omega r_{>}^{2}\right),
\end{aligned}
$$

where $\mathcal{M}_{a, b}(x)$ and $\mathcal{W}_{a, b}(x)$ are Whittaker functions [81].

\section{Momentum space LS equation and reaction amplitude in a harmonic oscillator trap}

The reaction amplitude in a harmonic trap can be defined by

$T_{\varepsilon}^{(h o)}(\mathbf{k})=-\int d \mathbf{r} e^{-i \mathbf{k} \cdot \mathbf{r}} \int d \mathbf{r}^{\prime \prime} V\left(\mathbf{r}^{\prime}, \mathbf{r}^{\prime \prime}\right) \psi_{\varepsilon}^{(h o)}\left(\mathbf{r}^{\prime \prime}\right)$,

and $T_{\varepsilon}^{(h o)}(\mathbf{k})$ satisfies the momentum space LS equation,

$T_{\varepsilon}^{(h o)}(\mathbf{k})=\int \frac{d \mathbf{k}^{\prime}}{(2 \pi)^{3}} \frac{d \mathbf{k}^{\prime \prime}}{(2 \pi)^{3}} \tilde{V}\left(\mathbf{k}, \mathbf{k}^{\prime}\right) \tilde{G}^{(h o)}\left(\mathbf{k}^{\prime}, \mathbf{k}^{\prime \prime} ; \varepsilon\right) T_{\varepsilon}^{(h o)}\left(\mathbf{k}^{\prime \prime}\right)$,

where $\tilde{V}$ and $\tilde{G}^{(h o)}$ are the Fourier transform of the interaction potential $V$ and Green's function $G^{(h o)}$ respectively. In a harmonic oscillator trap, rotation symmetry is intact, hence the angular momentum is still a good quantum number; the partial wave expansion of

$$
T_{\varepsilon}^{(h o)}(\mathbf{k})=\sum_{l m} T_{l}^{(h o)}(k) Y_{l m}(\hat{\mathbf{k}})
$$

and

$$
\tilde{G}^{(h o)}\left(\mathbf{k}, \mathbf{k}^{\prime} ; \varepsilon\right)=\sum_{l m} Y_{l m}(\hat{\mathbf{k}}) \tilde{G}_{l}^{(h o)}\left(k, k^{\prime} ; \varepsilon\right) Y_{l m}^{*}\left(\hat{\mathbf{k}}^{\prime}\right)
$$

thus yields

$$
\begin{aligned}
& T_{l}^{(h o)}(k) \\
& \quad=\int_{0}^{\infty} \frac{k^{\prime 2} d k^{\prime}}{(2 \pi)^{3}} \frac{k^{\prime \prime 2} d k^{\prime \prime}}{(2 \pi)^{3}} \tilde{V}_{l}\left(k, k^{\prime}\right) \tilde{G}_{l}^{(h o)}\left(k^{\prime}, k^{\prime \prime} ; \varepsilon\right) T_{l}^{(h o)}\left(k^{\prime \prime}\right) .
\end{aligned}
$$

The separable potential

$$
\tilde{V}_{l}\left(k, k^{\prime}\right)=\left(k k^{\prime}\right)^{l} V_{l}
$$

suggests that

$$
T_{l}^{(h o)}(k)=k^{l} t_{l}^{(h o)}
$$

hence the quantization condition under the assumption of a separable potential is given by

$$
\frac{1}{V_{l}}=\int_{0}^{\infty} \frac{k^{\prime 2} d k^{\prime}}{(2 \pi)^{3}} \frac{k^{\prime \prime 2} d k^{\prime \prime}}{(2 \pi)^{3}}\left(k^{\prime} k^{\prime \prime}\right)^{l} \tilde{G}_{l}^{(h o)}\left(k^{\prime}, k^{\prime \prime} ; \varepsilon\right)
$$

\section{Momentum space LS equation and scattering amplitude in infinite volume}

In infinite volume, the scattering amplitude is defined by

$T_{\varepsilon_{\infty}}^{(\infty)}(\mathbf{k}, \mathbf{q})=-\int d \mathbf{r} e^{-i \mathbf{k} \cdot \mathbf{r}} \int d \mathbf{r}^{\prime} V\left(\mathbf{r}, \mathbf{r}^{\prime}\right) \psi_{\varepsilon_{\infty}}^{(\infty)}\left(\mathbf{r}^{\prime}, \mathbf{q}\right)$,

and it satisfies the momentum space LS equation

$T_{\varepsilon_{\infty}}^{(\infty)}(\mathbf{k}, \mathbf{q})=-\tilde{V}(\mathbf{k}, \mathbf{q})+\int \frac{d \mathbf{k}^{\prime}}{(2 \pi)^{3}} \frac{\tilde{V}\left(\mathbf{k}, \mathbf{k}^{\prime}\right)}{\frac{\mathbf{k}^{\prime 2}}{2 \mu}-\frac{q^{2}}{2 \mu}} T_{\varepsilon_{\infty}}^{(\infty)}\left(\mathbf{k}^{\prime}, \mathbf{q}\right)$

The partial wave expansion

$$
T_{\varepsilon_{\infty}}^{(\infty)}(\mathbf{k}, \mathbf{q})=\sum_{l m} T_{l}^{(\infty)}(k, q) Y_{l m}(\hat{\mathbf{k}}) Y_{l m}^{*}(\hat{\mathbf{q}})
$$

yields 


$$
T_{l}^{(\infty)}(k, q)=-\tilde{V}_{l}(k, q)+\int_{0}^{\infty} \frac{k^{\prime 2} d k^{\prime}}{(2 \pi)^{3}} \frac{\tilde{V}_{l}\left(k, k^{\prime}\right)}{\frac{k^{\prime 2}}{2 \mu}-\frac{q^{2}}{2 \mu}} T_{l}^{(\infty)}\left(k^{\prime}, q\right) .
$$

The assumption of the separable potential again yields an analytic solution of scattering amplitude,

$$
T_{l}^{(\infty)}\left(q^{\prime}, q\right)=-\frac{\left(q^{\prime} q\right)^{L}}{\frac{1}{V_{l}}-\int_{0}^{\infty} \frac{k^{2} d k}{(2 \pi)^{3}} \frac{k^{2 l}}{2 \mu} \frac{k^{2}}{2 \mu}} .
$$

The on-shell partial wave scattering amplitudes $T_{l}^{(\infty)}(q, q)$, where $q=\sqrt{2 \mu \varepsilon_{\infty}}$, are usually parametrized by phase shift,

$$
T_{l}^{(\infty)}(q, q)=\frac{(4 \pi)^{2}}{2 \mu q} \frac{1}{\cot \delta_{l}(q)-i} .
$$

Therefore, a simple relation between $V_{L}$ and phase shift is obtained,

$$
\frac{1}{V_{l}}-\int_{0}^{\infty} \frac{k^{2} d k}{(2 \pi)^{3}} \frac{k^{2 l}}{\frac{k^{2}}{2 \mu}-\frac{q^{2}}{2 \mu}}=-\frac{2 \mu q^{2 l+1}}{(4 \pi)^{2}}\left[\cot \delta_{l}(q)-i\right] .
$$

\section{Quantization condition in a harmonic oscillator trap}

Combining Eqs. (C19) and (C13), we find

$$
\begin{gathered}
\int_{0}^{\infty} \frac{k^{2} d k}{(2 \pi)^{3}} \frac{k^{\prime 2} d k^{\prime}}{(2 \pi)^{3}}\left(k k^{\prime}\right)^{l} \tilde{G}_{l}^{(h o)}\left(k, k^{\prime} ; \varepsilon\right)-\int_{0}^{\infty} \frac{k^{2} d k}{(2 \pi)^{3}} \frac{k^{2 l}}{\frac{k^{2}}{2 \mu}-\frac{q^{2}}{2 \mu}} \\
=-\frac{2 \mu q^{2 l+1}}{(4 \pi)^{2}}\left[\cot \delta_{l}(q)-i\right] .
\end{gathered}
$$

Using an asymptotic form of the spherical Bessel function,

$$
j_{l}(k r) \stackrel{r \rightarrow 0}{\longrightarrow} \frac{\sqrt{\pi}(k r)^{l}}{2^{l+1} \Gamma\left(l+\frac{3}{2}\right)},
$$

one can easily prove that

$$
\begin{gathered}
\int_{0}^{\infty} \frac{k^{2} d k}{(2 \pi)^{3}} \frac{k^{\prime 2} d k^{\prime}}{(2 \pi)^{3}}\left(k k^{\prime}\right)^{l} \tilde{G}_{l}^{(h o)}\left(k, k^{\prime} ; \varepsilon\right) \\
=\left.\frac{1}{(4 \pi)^{2}} \frac{2^{2 l+2} \Gamma^{2}\left(l+\frac{3}{2}\right)}{\pi} \frac{G_{l}^{(h o)}\left(r, r^{\prime} ; \varepsilon\right)}{\left(r r^{\prime}\right)^{l}}\right|_{r, r^{\prime} \rightarrow 0},
\end{gathered}
$$

and

$$
\int_{0}^{\infty} \frac{k^{2} d k}{(2 \pi)^{3} \frac{k^{2 l}}{2 \mu}-\frac{q^{2}}{2 \mu}}=\left.\frac{1}{(4 \pi)^{2}} \frac{2^{2 l+2} \Gamma^{2}\left(l+\frac{3}{2}\right)}{\pi} \frac{G_{l}^{(\infty)}\left(r, r^{\prime} ; q\right)}{\left(r r^{\prime}\right)^{l}}\right|_{r, r^{\prime} \rightarrow 0},
$$

where the analytic expression of $G_{l}^{(h o)}\left(r, r^{\prime} ; \varepsilon\right)$ and $G_{l}^{(\infty)}\left(r, r^{\prime} ; q\right)$ are given in Eqs. (C7) and (B24) respectively. Also using the asymptotic form of the harmonic oscillator trap Green's function,

$$
\begin{gathered}
\left.\frac{2^{2 l+2} \Gamma^{2}\left(l+\frac{3}{2}\right)}{\pi} \frac{G_{l}^{(h o)}\left(r, r^{\prime} ; \varepsilon\right)}{\left(r r^{\prime}\right)^{l}}\right|_{r, r^{\prime} \rightarrow 0} \\
=-\frac{(\mu \omega)^{l+\frac{3}{2}}}{\omega} 2^{2 l+2}(-1)^{l+1} \frac{\Gamma\left(\frac{3}{4}+\frac{l}{2}-\frac{\varepsilon}{2 \omega}\right)}{\Gamma\left(\frac{1}{4}-\frac{l}{2}-\frac{\epsilon_{n}}{2 \omega}\right)} \\
-\left.\frac{2^{2 l+1} \Gamma\left(l+\frac{1}{2}\right) \Gamma\left(l+\frac{3}{2}\right)}{\pi} \frac{2 \mu}{r^{2 l+1}}\right|_{r \rightarrow 0},
\end{gathered}
$$

and asymptotic form of $G_{l}^{(\infty)}\left(r, r^{\prime} ; q\right)$ given in Eq. (B30), the UV divergence cancels out explicitly in Eq. (C20), and the quantization condition is thus reduced to the BERW formula,

$\cot \delta_{l}(q)-(-1)^{l+1}\left(\frac{4 \mu \omega}{q^{2}}\right)^{l+\frac{1}{2}} \frac{\Gamma\left(\frac{3}{4}+\frac{l}{2}-\frac{\varepsilon}{2 \omega}\right)}{\Gamma\left(\frac{1}{4}-\frac{l}{2}-\frac{\varepsilon}{2 \omega}\right)}=0$,

where $q$ and $\varepsilon$ are associated by

$$
\frac{q^{2}}{2 \mu}+\frac{\mathbf{P}^{2}}{2 M}=\varepsilon+\omega\left(n+\frac{3}{2}\right)
$$

[1] M. Lüscher, Nucl. Phys. B354, 531 (1991).

[2] K. Rummukainen and S. A. Gottlieb, Nucl. Phys. B450, 397 (1995).

[3] N. H. Christ, C. Kim, and T. Yamazaki, Phys. Rev. D 72, 114506 (2005).

[4] V. Bernard, M. Lage, U.-G. Meißner, and A. Rusetsky, J. High Energy Phys. 08 (2008) 024.
[5] S. He, X. Feng, and C. Liu, J. High Energy Phys. 07 (2005) 011.

[6] M. Lage, U.-G. Meißner, and A. Rusetsky, Phys. Lett. B 681, 439 (2009).

[7] M. Döring, U.-G. Meißner, E. Oset, and A. Rusetsky, Eur. Phys. J. A 47, 139 (2011).

[8] P. Guo, J. Dudek, R. Edwards, and A. P. Szczepaniak, Phys. Rev. D 88, 014501 (2013). 
[9] P. Guo, Phys. Rev. D 88, 014507 (2013).

[10] S. Kreuzer and H. W. Hammer, Phys. Lett. B 673, 260 (2009).

[11] K. Polejaeva and A. Rusetsky, Eur. Phys. J. A 48, 67 (2012).

[12] M. T. Hansen and S. R. Sharpe, Phys. Rev. D 90, 116003 (2014).

[13] U.-G. Meißner, G. Ríos, and A. Rusetsky, Phys. Rev. Lett. 114, 091602 (2015); 117, 069902(E) (2016).

[14] M. Mai and M. Döring, Eur. Phys. J. A 53, 240 (2017).

[15] M. Mai and M. Döring, Phys. Rev. Lett. 122, 062503 (2019).

[16] M. Döring, H. W. Hammer, M. Mai, J. Y. Pang, A. Rusetsky, and J. Wu, Phys. Rev. D 97, 114508 (2018).

[17] P. Guo, Phys. Rev. D 95, 054508 (2017).

[18] P. Guo and V. Gasparian, Phys. Lett. B 774, 441 (2017).

[19] P. Guo and V. Gasparian, Phys. Rev. D 97, 014504 (2018).

[20] P. Guo and T. Morris, Phys. Rev. D 99, 014501 (2019).

[21] T. D. Blanton, F. Romero-López, and S. R. Sharpe, Phys. Rev. Lett. 124, 032001 (2020).

[22] M. Mai, M. Döring, C. Culver, and A. Alexandru, Phys. Rev. D 101, 054510 (2020).

[23] P. Guo, M. Döring, and A. P. Szczepaniak, Phys. Rev. D 98, 094502 (2018).

[24] P. Guo, Phys. Lett. B 804, 135370 (2020).

[25] P. Guo and M. Döring, Phys. Rev. D 101, 034501 (2020).

[26] P. Guo, Phys. Rev. D 101, 054512 (2020).

[27] P. Guo and B. Long, Phys. Rev. D 101, 094510 (2020).

[28] P. Guo, arXiv:2007.04473.

[29] P. Guo and B. Long, Phys. Rev. D 102, 074508 (2020).

[30] P. Guo, Phys. Rev. D 102, 054514 (2020).

[31] S. Aoki et al. (CP-PACS Collaboration), Phys. Rev. D 76, 094506 (2007).

[32] X. Feng, K. Jansen, and D. B. Renner, Phys. Rev. D 83, 094505 (2011).

[33] C. B. Lang, D. Mohler, S. Prelovsek, and M. Vidmar, Phys. Rev. D 84, 054503 (2011); 89, 059903(E) (2014).

[34] S. Aoki et al. (CS Collaboration), Phys. Rev. D 84, 094505 (2011).

[35] J. J. Dudek, R. G. Edwards, and C. E. Thomas, Phys. Rev. D 86, 034031 (2012).

[36] D. J. Wilson, J. J. Dudek, R. G. Edwards, and C. E. Thomas, Phys. Rev. D 91, 054008 (2015).

[37] S. R. Beane, W. Detmold, T. C. Luu, K. Orginos, M. J. Savage, and A. Torok, Phys. Rev. Lett. 100, 082004 (2008).

[38] W. Detmold, M. J. Savage, A. Torok, S. R. Beane, T. C. Luu, K. Orginos, and A. Parreno, Phys. Rev. D 78, 014507 (2008).

[39] B. Hörz and A. Hanlon, Phys. Rev. Lett. 123, 142002 (2019).

[40] R. Brett, C. Culver, M. Mai, A. Alexandru, M. Döring, and F. X. Lee, arXiv:2101.06144.

[41] A. Alexandru, R. Brett, C. Culver, M. Döring, D. Guo, F. X. Lee, and M. Mai, Phys. Rev. D 102, 114523 (2020).

[42] T. Busch, B.-G. Englert, K. Rzażewski, and M. Wilkens, Found. Phys. 28, 549 (1998).

[43] I. Stetcu, B. Barrett, U. van Kolck, and J. Vary, Phys. Rev. A 76, 063613 (2007).

[44] I. Stetcu, J. Rotureau, B. Barrett, and U. van Kolck, Ann. Phys. (Amsterdam) 325, 1644 (2010).
[45] J. Rotureau, I. Stetcu, B. Barrett, M. Birse, and U. van Kolck, Phys. Rev. A 82, 032711 (2010).

[46] J. Rotureau, I. Stetcu, B. Barrett, and U. van Kolck, Phys. Rev. C 85, 034003 (2012).

[47] T. Luu, M. J. Savage, A. Schwenk, and J. P. Vary, Phys. Rev. C 82, 034003 (2010).

[48] C.-J. Yang, Phys. Rev. C 94, 064004 (2016).

[49] C. W. Johnson et al., J. Phys. G 47, 123001 (2020).

[50] X. Zhang, Phys. Rev. C 101, 051602 (2020).

[51] X. Zhang, S. Stroberg, P. Navrátil, C. Gwak, J. Melendez, R. Furnstahl, and J. Holt, Phys. Rev. Lett. 125, 112503 (2020).

[52] S.-K. Yip, Phys. Rev. A 78, 013612 (2008).

[53] A. Suzuki, Y. Liang, and R. K. Bhaduri, Phys. Rev. A 80, 033601 (2009).

[54] S. Elhatisari, D. Lee, U.-G. Meißner, and G. Rupak, Eur. Phys. J. A 52, 174 (2016).

[55] A. Rokash, M. Pine, S. Elhatisari, D. Lee, E. Epelbaum, and H. Krebs, Phys. Rev. C 92, 054612 (2015).

[56] E. Epelbaum and U.-G. Meissner, Phys. Lett. B 461, 287 (1999); 467, 308(E) (1999).

[57] X. Kong and F. Ravndal, Nucl. Phys. A665, 137 (2000).

[58] S. R. Beane et al., Phys. Rev. D 103, 054504 (2021).

[59] S. R. Beane and M. J. Savage, Phys. Rev. D 90, 074511 (2014).

[60] G. Stellin and U.-G. Meißner, Eur. Phys. J. A 57, 26 (2021).

[61] R. Horsley et al., J. Phys. G 43, 10 LT02 (2016).

[62] R. Horsley et al., J. High Energy Phys. 04 (2016) 093.

[63] R. Horsley et al. (CSSM, QCDSF, and UKQCD Collaborations), J. Phys. G 46, 115004 (2019).

[64] V. Lubicz, G. Martinelli, C. T. Sachrajda, F. Sanfilippo, S. Simula, and N. Tantalo, Phys. Rev. D 95, 034504 (2017).

[65] T. Blum, N. Christ, M. Hayakawa, T. Izubuchi, L. Jin, C. Jung, and C. Lehner, Phys. Rev. D 96, 034515 (2017).

[66] Z. Davoudi and M. J. Savage, Phys. Rev. D 90, 054503 (2014).

[67] M. G. Endres, A. Shindler, B. C. Tiburzi, and A. Walker-Loud, Phys. Rev. Lett. 117, 072002 (2016).

[68] D. J. Thouless, M. Kohmoto, M. P. Nightingale, and M. den Nijs, Phys. Rev. Lett. 49, 405 (1982).

[69] Y. Hatsugai, Phys. Rev. Lett. 71, 3697 (1993).

[70] Y. Hatsugai, J. Phys. Condens. Matter 9, 2507 (1997).

[71] W. Detmold and M. J. Savage, Nucl. Phys. A743, 170 (2004).

[72] W. Detmold, Phys. Rev. D 71, 054506 (2005).

[73] W. Detmold, B. C. Tiburzi, and A. Walker-Loud, Proc. Sci., LATTICE2008 (2008) 147 [arXiv:0809.0721].

[74] W. Detmold, B. C. Tiburzi, and A. Walker-Loud, eCONF C0906083, 03 (2009).

[75] E. Brown, Phys. Rev. 133, A1038 (1964).

[76] J. Zak, Phys. Rev. 134, A1602 (1964).

[77] D. Yoshioka, The Quantum Hall Effect, Physics and Astronomy Online Library (Springer, New York, 2002).

[78] T. Ueta, J. Phys. Soc. Jpn. 61, 4314 (1992).

[79] A. K. Rajagopal and J. C. Ryan, Phys. Rev. B 44, 10280 (1991).

[80] S. Blinder, J. Math. Phys. (N.Y.) 25, 905 (1984).

[81] E. T. Whittaker and G. N. Watson, A Course of Modern Analysis, 4th ed., Cambridge Mathematical Library (Cambridge University Press, Cambridge, England, 1996). 